\title{
Comparative Study of Small-Signal Stability under Weak AC System Integration for Different VSCs
}

\author{
Xiaojun Lu, Wang Xiang, Member, IEEE, Weixing Lin, Member, IEEE, Jinyu Wen, Member, IEEE
}

\begin{abstract}
Voltage source converters (VSCs) with self-commutation ability are suitable to interconnect weak AC systems. This paper conducts a comparative study of the small-signal stability characteristics for three typical VSCs, namely, the two-level VSCs (TL-VSCs), the half-bridge modular multilevel converters (HBMMCs) and the hybrid MMCs (HY-MMCs), under weak AC system integration with special consideration on both inverter and rectifier operation. The frequency responses based on impedance models are compared using the frequency-domain analysis. The oscillation frequencies and mainly participated state variables of the unstable modes are compared using root locus and participation factor analysis in time-domain. Proper parameter retuning approaches for stability enhancement are proposed. The above analysis is adequately validated by electromagnetic simulations.
\end{abstract}

Index Terms-Small-signal model, small-signal stability, modular multilevel converter, weak AC system, HVDC transmission.

\section{NOMENCLATURE}

\section{Acronyms}

VSC

MMC

IGBT

TL-VSC

voltage source converter

modular multilevel converter

insulated gate bipolar transistor

HB-MMC

two-level voltage source converter

HY-MMC

half-bridge modular multilevel converter

HBSM

FBSM

OHL

SCR

ACC

$\mathrm{DCC}$

CCSC

PLL

$\mathrm{PF}$

PCC

CCS

CVS

hybrid modular multilevel converter

half-bridge sub-module

full-bridge sub-module

overhead line

short circuit ratio

AC current controller

DC current controller

circulating current suppression controller

phase lock loop

participation factor

point of common coupling

controlled current source

controlled voltage source

\section{Subscripts}

$x, y$

axis of the rotating frame synchronized with PCC voltage

$d, q$ axis of the rotating frame synchronized with the measured PCC voltage by PLL

\author{
pu per unit \\ fil filtered signals \\ ref reference values
}

\section{Variables}

$u_{s}$

$P_{a c}$

$Q$

$u_{d c}$

$i_{d c}$

$i$

$u_{v}$

$u_{p}$

$m$

$m_{p}, m_{n}$

$i_{u p}, i_{d n}$

$u_{c p}^{\Sigma}, u_{c n}^{\Sigma}$

$M_{x}, M_{y} /$

$M_{d}, M_{q}$

$M_{x 2}, M_{y 2} /$

$M_{d 2}, M_{q 2}$

$M_{d c}$

$u_{c x 2}^{\Sigma}, u_{c y 2}^{\Sigma}$

$u_{c x}^{\Sigma}, u_{c y}^{\Sigma}$

$u_{c 0}^{\Sigma}$

$u_{c, a v g}$

$u_{v x}, u_{v y}$

$i_{x}, i_{y} / i_{d}, i_{q}$

$i_{\text {diff }}$

$i_{\text {diffo }}$

$i_{\text {diff } x 2}, i_{\text {diffy } 2}$

$x_{P}, x_{Q}, x_{I d}, x_{I q}$

$x_{P d c}, x_{I d c}$

\section{Parameters}

$R_{s} / L_{s}$

$K_{T}$

$R_{T} / L_{T}$

$C_{d c}$

PCC voltage lower arm $x y / d q$ frame

frame frame

$u_{v}$ in $x y$ frame

$i$ in $x y / d q$ frame
AC system voltage

AC-side active power of converters

reactive power of converters

DC terminal voltage of VSCs

DC terminal current of VSCs

AC-side current of VSCs

AC-side voltage of VSCs

modulation signal of VSCs

modulation signals for upper and lower arm upper and lower arm current

the total capacitor voltage of the upper and

fundamental-frequency modulation signal in

second harmonic modulation signal in $x y / d q$

DC modulation signal

second harmonic of $u_{c p}^{\Sigma}$ in $x y$ frame

fundamental-frequency part of $u_{c p}^{\Sigma}$ in $x y$

DC component of $u_{c p}^{\Sigma}$

average capacitor voltage, equal to $u_{c 0}^{\Sigma}$

circulating current

DC component of $i_{\text {diff }}$

second harmonic of $i_{\text {diff }}$ in $x y$ frame

state variables in ACC outer and inner loop

state variables in DCC outer and inner loop

equivalent $\mathrm{AC}$ system resistance/inductance

turn-ratio of the AC transformer

$\mathrm{AC}$ transformer resistance/inductance

lumped capacitance of TL-VSC

\footnotetext{
${ }^{1}$ This work is sponsored by the Joint Funds of the National Natural Science Foundation of China (U1766211), the National Natural Science Foundation of China (51907068) and the Key Research and Development Program of Shaanxi (2017ZDXM-GY-135). (Corresponding author: Wang Xiang)

$\mathrm{X}$. Lu, and J. Wen are with the State Key Laboratory of Advanced Electromagnetic Engineering and Technology, Huazhong University of Science
}

and Technology, Wuhan 430074, China. (e-mail:luxiaojun1212@ foxmail.com, jinyu.wen @ hust.edu.cn).

W. Xiang is with the Department of Electronic and Electrical Engineering, University of Strathclyde, Glasgow, G1 1XW, U.K. (e-mail: xiangwang1003@ foxmail.com).

W. Lin is with the TBEA SunOasis Co,. Ltd, Urumuchi 830011, Xinjiang, China. (e-mail: weixinglin@foxmail.com). 


\begin{tabular}{|c|c|}
\hline$C_{\text {sub }}$ & capacitance of sub-modules in MMC \\
\hline$R_{\text {arm }}$ & equivalent resistance of MMC per arm \\
\hline$L_{\text {arm }}$ & Arm inductance of MMC \\
\hline$L_{a c}$ & AC inductance for TL-VSC \\
\hline$N_{\text {sub }}$ & $\begin{array}{l}\text { total number of sub-modules in one MMC } \\
\text { arm }\end{array}$ \\
\hline$K_{P O} / K_{P I}$ & proportional gain for ACC outer/inner loop \\
\hline$K_{I O} / K_{I I}$ & integration gain for ACC outer/inner loop \\
\hline & feedforward gain for AC current in ACC \\
\hline$K_{P O d d} / K_{P I d c}$ & proportional gain for DCC outer/inner loop \\
\hline$K_{I O d d} / K_{I I d c}$ & integration gain for DCC outer/inner loop \\
\hline$K_{U d c s}$ & feed-forward gain of DC voltage \\
\hline$K_{P p l l} / K_{\text {Ipll }}$ & proportional and integration gain of PLL \\
\hline$K_{P C} / K_{I C}$ & proportional and integration gain for CCSC \\
\hline
\end{tabular}

VSCs are self-commutated, which are suitable to interconnect renewable generation systems or other weak AC systems. Various topologies of VSCs have been applied in commercial applications, such as TL-VSCs [1], HB-MMCs [2] and HY-MMCs [3].

The TL-VSCs with IGBTs in series connection are adopted for integrating wind power or interconnecting asynchronous AC systems, with lumped DC capacitors installed at the DCside to support the DC link voltage. Restricted by the capacity and voltage withstand ability, TL-VSCs are mainly adopted in applications with low DC voltage and power ratings [1]. Driven by the need for high-voltage bulk-power transmission, MMCs based on HBSMs are proposed in [2]. The distributed capacitors in SMs relieve the requirement for the simultaneous triggering of IGBTs, making it easier to construct high DC voltage transmission. However, both TL-VSCs and HB-MMCs are vulnerable to DC faults [4][5], limiting their application in OHL transmission systems. The HY-MMC is proposed in [3], with FBSMs and HBSMs mixed in the phase arms. The negative insertion of the FBSMs endows HY-MMC with the ability to control its DC output voltage independently of the AC voltage. By optimally designing the sub-module ratio and the active fault current control strategy, HY-MMC can ride through DC faults without interruption [6][7].

The self-commutation ability of VSCs makes them applicable to the weak AC grid connection. The grid-side converter for the wind power generation system normally adopts TL-VSCs, which are usually integrated through longdistance $\mathrm{AC}$ transmission lines with relatively large reactance, resulting in a weak AC system integration situation. Under such conditions, small-signal stability of TL-VSCs may be worsened because of the undesired interaction among different control units [8]-[10] or the negative resistance effect of the AC output impedance [12][13]. While for practical MMC projects, such as a $\pm 350 \mathrm{kV} / 1000 \mathrm{MW}$ back-to-back HVDC HB-MMC project [14], or a $\pm 800 \mathrm{kV}$ hybrid three-terminal OHL-HVDC project employing HY-MMCs [15], the converter is normally connected to a strong AC system. But the SCR of the AC system can be varied due to the variation of the seasonal loads or the change of the grid structure by the scheduled maintenance or occasional AC faults. Therefore, MMCs may be forced to be connected with a weak AC system, which may cause smallsignal stability problems under usual operation points and regular control modes, such as high-frequency oscillation in the AC voltage [14][15], or a wide-band oscillation in the transmitted power as reported in [16].

The above-mentioned instability has been separately researched employing the time-domain modal analysis or the frequency-domain impedance analysis [8]-[16]. However, the instability characteristic of these three topologies under weak $\mathrm{AC}$ system integration has not been adequately compared. The small-signal dynamics for the TL-VSCs and HB-MMCs are compared in [17], which shows that HB-MMCs produce more complex interaction patterns due to the internal dynamics. Nevertheless, for the stability issue under weak AC system integration, whether the internal dynamics of MMCs may affect the stability characteristics still needs to be investigated.

Existing publications mainly focus on one specific operation state for a single terminal VSC under constant AC voltage control mode. In practical MMC-HVDC projects, the reactive power control instead of the AC voltage is conventionally adopted. As mentioned before, the strength of the AC system may be suddenly weakened due to the tripping of $\mathrm{AC}$ transmission lines. The stability characteristics of VSCs under reactive power control needs to be investigated for a weak system connection. Moreover, some specially designed control strategies for HB-MMCs or HY-MMCs, such as the CCSC or the DC fault-ride-through control, may also affect the smallsignal stability and cause some difference. The comparative study needs to be conducted to obtain an in-depth understanding.

The contributions of this paper are as follows:

1) In this paper, the stability analysis with special consideration on both rectifier and inverter operation is carried out for VSCs under constant reactive power and DC voltage control, which is a more general control strategy for high-power MMC-HVDC projects.

2) The stability comparison of TL-VSC, HB-MMC, and HYMMC is conducted for the first time under weak AC system integration, emphasizing the instability caused by excessive rectified or inverted active power.

3) Both single-terminal-based frequency-domain impedance analysis and point-to-point-based time-domain modal analysis have been employed, and the results are compared. Detailed dynamics of the control system including PLL, sampling filters and various current controllers are modeled in the impedance and state-space small-signal models.

4) Methods of retuning control parameters, including PLL, ACC, and DCC, are summarized to improve stability for different operation states.

The paper is organized as follows. A brief derivation of the dynamic models for the three VSCs is displayed in Section II. The single-terminal-based impedance analysis is presented in Section III. Section IV demonstrates the root locus and PF analysis with increasing the active power penetration based on 
a typical point-to-point system. The stability analysis results are validated by electromagnetic simulations in Section V. The improvement of stability by parameter adjustment is analyzed and validated in Section VI. The conclusion is finally drawn in Section VII.

\section{DYNAMIC MODELS}

The topologies for TL-VSC, HB-MMC, and HY-MMC are depicted in Fig. 1. The AC-side circuits of the three VSCs are the same. The parameters are listed in the Appendix.

\section{A. Electrical system model}

\section{1) The AC-side equivalent circuits}

Based on the equivalent circuit shown in Fig. 1 (a), the dynamics of the AC current $i$ can be obtained by applying KVL:

$$
u_{s} / K_{T}=R_{t o t} i+L_{t o t} \mathrm{~d} i / \mathrm{d} t+u_{v}
$$

where $R_{t o t}=\left(R_{s}+R_{T}\right) / K_{T}^{2}$. For HB/HY-MMC, $L_{t o t}=\left(L_{s}+L_{T}\right) / K_{T}^{2}$; for TL-VSC, $L_{t o t}=L_{a c}+\left(L_{s}+L_{T}\right) / K_{T}^{2}$.

For TL-VSCs, $u_{v}$ can be linked to $u_{d c}$ by the modulation signal $m[8]$ :

$$
u_{v}=m u_{d c} / 2
$$

Define the rotating frame $x y$ which is synchronized with the PCC voltage $u_{p}$, and $u_{v}$ can be expressed in $x y$ frame as:

$$
\begin{aligned}
& u_{v x}=M_{x} u_{d c} / 2 \\
& u_{v y}=M_{y} u_{d c} / 2
\end{aligned}
$$

While for HB-MMC or HY-MMC, $u_{v}$ is associated with the upper or lower arm output voltage [20]:

$$
\begin{aligned}
& u_{v}=u_{d c} / 2+R_{a r m} i_{u p}+L_{a r m} \mathrm{~d} i_{u p} / \mathrm{d} t-m_{p} u_{c p}^{\Sigma} \\
& u_{v}=-u_{d c} / 2+R_{a r m} i_{d n}+L_{a r m} \mathrm{~d} i_{d n} / \mathrm{d} t+m_{n} u_{c n}^{\Sigma}
\end{aligned}
$$

By adding up (5) and (6), we can obtain:

$$
u_{v}=\frac{R_{a r m}}{2} i+\frac{L_{a r m}}{2} \frac{\mathrm{d} i}{\mathrm{~d} t}+\underbrace{\left(m_{n} u_{c n}^{\Sigma}-m_{p} u_{c p}^{\Sigma}\right) / 2}_{e_{v}}
$$

The last item in (7) is defined as the internal AC output voltage of MMC $e_{v}$. Similarly, $e_{v}$ can be expressed in $x y$ frame:

$$
e_{v x}=\left(\frac{m_{n} u_{c n}^{\Sigma}-m_{p} u_{c p}^{\Sigma}}{2}\right)_{x}, e_{v y}=\left(\frac{m_{n} u_{c n}^{\Sigma}-m_{p} u_{c p}^{\Sigma}}{2}\right)_{y}
$$

By exploring the $x$ and $y$ components of the items in (8), we can obtain [18]:

$$
\left[\begin{array}{l}
e_{v x} \\
e_{v y}
\end{array}\right]=\left[\begin{array}{cccc}
\frac{M_{x}}{4} \frac{M_{y}}{4} \frac{M_{x 2}-2 M_{d c}}{4} & \frac{M_{y 2}}{4} & \frac{M_{x}}{2} \\
\frac{-M_{y}}{4} \frac{M_{x}}{4} & \frac{M_{y 2}}{4} & -\frac{M_{x 2}+2 M_{d c}}{4} & \frac{M_{y}}{2}
\end{array}\right] \mathbf{u}_{\mathbf{c}}^{\Sigma}
$$

where $\mathbf{u}_{\mathbf{c}}^{\Sigma}=\left[u_{c x 2}^{\Sigma}, u_{c y 2}^{\Sigma}, u_{c x}^{\Sigma}, u_{c y}^{\Sigma}, u_{c 0}^{\Sigma}\right]^{T} . M_{d c}$ is usually set to be constant 1 in HB-MMCs. On the contrary, $M_{d c}$ can be flexibly regulated in HY-MMCs due to the negative insertion of the submodules [18].

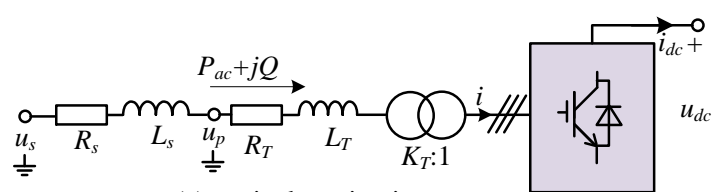

(a) equivalent circuit

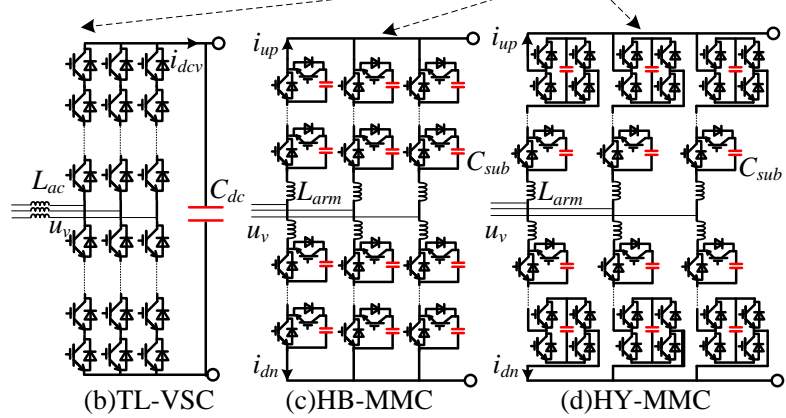

Fig. 1. Equivalent circuit and topologies of the three VSCs.

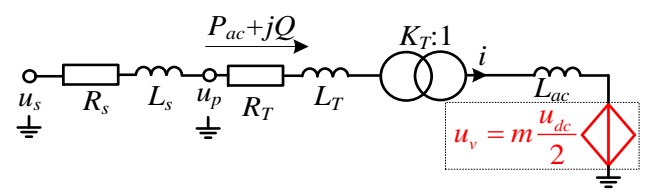

(a) AC-side equivalent circuit for TL-VSCs

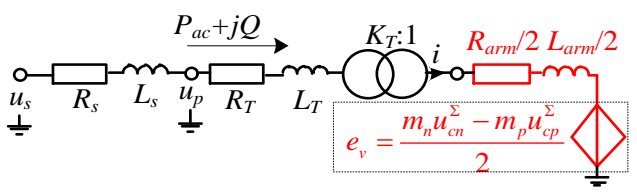

(b) AC-side equivalent circuit for HB-MMCs or HY-MMCs

Fig. 2. AC-side equivalent circuits.

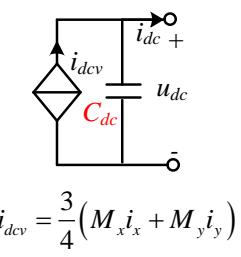

(a) TL-VSC

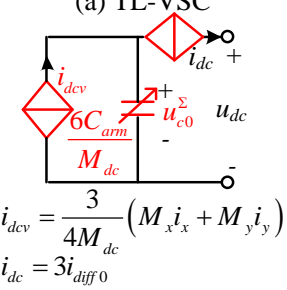

(c)HY-MMC (CCS type)

Fig. 3. DC-side equivalent circuits.

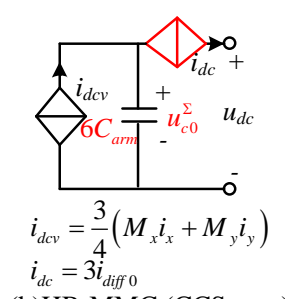

(b)HB-MMC (CCS type)

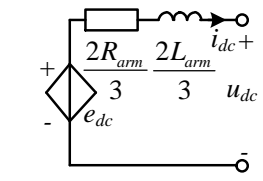

$e_{d c}:$ Equ. 16 or Equ. 17 $i_{d c}^{d c}=3 i_{d i f f 0}$

(d)HB/HY-MMC (CVS type)
Based on the above derivation, the $\mathrm{AC}$-side equivalent circuits for TL-VSC, HB-MMC, and HY-MMC are depicted in Fig. 2. The expressions of the AC-side controlled voltages are similar, which are the productions of the modulation signal and the capacitor voltage.

2) The DC-side equivalent circuit

For TL-VSC, the dynamics of capacitor voltage $u_{d c}$ can be 
described as:

$$
C_{d c} \frac{\mathrm{d} u_{d c}}{\mathrm{~d} t}=i_{d c}-i_{d c v}
$$

The DC current $i_{d c v}$ is generally obtained by considering the active power balance between the $\mathrm{AC}$ and $\mathrm{DC}$ side:

$$
1.5\left(u_{v x} i_{x}+u_{v y} i_{y}\right)=u_{d c} i_{d c v}
$$

Substitute (3) and (4) into (11), and we can obtain:

$$
i_{d c v}=0.75\left(M_{x} i_{x}+M_{y} i_{y}\right)
$$

Based on the above derivation, the DC-side equivalent circuit for TL-VSC is depicted in Fig. 3 (a). The CCS is generated by the production of the modulation signals and the AC current, and $i_{d c}$ is provided from the DC network.

For HB-MMC or HY-MMC, the dynamics of the capacitor voltage can be described as [20]:

$$
C_{a r m} \frac{\mathrm{d} u_{c p}^{\Sigma}}{\mathrm{d} t}=-m_{p} i_{u p}
$$

where $C_{a r m}=C_{s u b} / N_{s u b}$.

If we expand the expressions of the variables in (13) under the rotating $x y$ frame, and retain only the DC components, we can get the dynamics of the DC total capacitor voltage. For HBMMC, we have[19]:

$$
6 C_{a r m} \frac{\mathrm{d} u_{c 0}^{\Sigma}}{\mathrm{d} t}=\underbrace{0.75\left(M_{x} i_{x}+M_{y} i_{y}\right)}_{i_{d c v}}-3 i_{d i f f 0}
$$

The first term on the right side of equation (14) is represented by $i_{d c v}$, and the second item is denoted as $i_{d c}$. Then the DC-side equivalent circuit based on CCS for HB-MMC is depicted in Fig. 3 (b). Different from TL-VSC, $i_{d c}$ in HB-MMC is decided by its state variable $i_{d i f f 0}$ instead of the DC network. The DC voltage of the equivalent capacitance $6 C_{a r m}$ is equal to $u_{c 0}^{\Sigma}$, instead of the DC terminal voltage.

While for HY-MMC, due to the variable $M_{d c}$, the dynamic expression for $u_{c 0}^{\Sigma}$ is different from that of HB-MMC:

$$
\frac{6 C_{a r m}}{M_{d c}} \frac{\mathrm{d} u_{c 0}^{\Sigma}}{\mathrm{d} t}=\underbrace{\frac{3}{4 M_{d c}}\left(M_{x} i_{x}+M_{y} i_{y}\right)}_{i_{d c v}}-3 i_{d i f f 0}
$$

As shown in Fig. 3 (c), the expressions for the equivalent capacitance and the CCS $i_{d c v}$ in HY-MMC are different from those in HB-MMC, both of which are related to $M_{d c}$.

The voltages across the CCS in Fig. 3 (b) and (c) remain to be solved, which makes it difficult to analyze. Instead, we can obtain the dynamic equation for $i_{d c}$ by subtracting (5) and (6), and retaining only the DC component. For HB-MMC, we have[19][20]:

$$
\begin{aligned}
& \frac{2 L_{a r m}}{3} \frac{\mathrm{d} i_{d c}}{\mathrm{~d} t}=\frac{2 R_{a r m}}{3} i_{d c}+u_{d c}+ \\
& \underbrace{u_{c 0}^{\Sigma}-\frac{1}{2}\left(M_{x} u_{c x}^{\Sigma}+M_{y} u_{c y}^{\Sigma}+M_{x 2} u_{c x 2}^{\Sigma}+M_{y 2} u_{c y 2}^{\Sigma}\right)}_{e_{d c}}
\end{aligned}
$$

For HY-MMC, we have[18]:

$$
\begin{aligned}
& \frac{2 L_{a r m}}{3} \frac{\mathrm{d} i_{d c}}{\mathrm{~d} t}=\frac{2 R_{a r m}}{3} i_{d c}+u_{d c}+ \\
& \underbrace{M_{d c} u_{c 0}^{\Sigma}-\frac{1}{2}\left(M_{x} u_{c x}^{\Sigma}+M_{y} u_{c y}^{\Sigma}+M_{x 2} u_{c x 2}^{\Sigma}+M_{y 2} u_{c y 2}^{\Sigma}\right)}_{e_{d c}}
\end{aligned}
$$

The equivalent CVS $e_{d c}$ for HB-MMC and HY-MMC is respectively given in (16) and (17), which is defined as the internal DC output voltage. According to (16) and (17), we can obtain the CVS-based equivalent circuit for HB-MMC or HYMMC, as shown in Fig. 3 (d).

\section{B. Control system model}

The classical vector-based current control for the three topologies are depicted in Fig. 4. The TL-VSC only adopts ACC, and the control target $\alpha$ for the $d$ axis outer loop can be selected as either $P_{a c}$ or $u_{d c}$. In comparison, HB-MMC additionally adopts CCSC. The DC current for TL-VSC or HB$\mathrm{MMC}$ is constrained by implicit AC/DC active power balance with the relatively constant $\mathrm{DC}$ voltage.

For HY-MMC, an extra DCC can be employed to actively control the DC fault current[7]. The outer loop control target $\alpha$ for the $d$ axis is fixed as the average capacitor voltage $u_{c, a v g}$,

\begin{tabular}{|c|c|c|c|c|}
\hline \multicolumn{2}{|c|}{ State variables (order) } & TL-VSC & HB-MMC & "HY-MMC \\
\hline \multicolumn{2}{|c|}{ AC current } & $i_{x}, i_{y}$ & $i_{x}, i_{y}$ & $i_{x}, i_{y}$ \\
\hline \multirow{3}{*}{$\begin{array}{l}\text { VSC } \\
\text { electric } \\
\text { system }\end{array}$} & Capacitor voltage & $u_{d c}$ & $u_{c}^{\Sigma}$ & $\mathbf{u}_{\mathrm{c}}^{\Sigma}$ \\
\hline & Circulating current & - & $i_{d i f f x 2}, i_{d i f f y 2}$ & $i_{\text {diff } x 2}, i_{\text {diffy } 2}$ \\
\hline & DC current & - & $i_{\text {diffo }}$ & $i_{\text {diffo }}$ \\
\hline \multirow{4}{*}{$\begin{array}{c}\text { VSC } \\
\text { control } \\
\text { system }\end{array}$} & $\begin{array}{c}\mathrm{ACC} \\
\mathrm{CCSC}\end{array}$ & $\begin{array}{c}x_{P}, x_{I d}, x_{Q}, x_{I q} \\
-\end{array}$ & $\begin{array}{c}x_{P}, x_{I d}, x_{Q}, x_{I q} \\
\text { (2) }\end{array}$ & $\begin{array}{c}x_{P}, x_{I d}, x_{Q}, x_{I q} \\
\text { (2) }\end{array}$ \\
\hline & DCC & 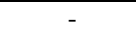 & - & $x_{P d c}, x_{I d c}$ \\
\hline & PLL & (2) & (2) & (2) \\
\hline & Signal filters & (14) & (18) & (22) \\
\hline \multicolumn{2}{|c|}{ Total order for single converter } & 21 & 34 & 40 \\
\hline
\end{tabular}
which is equal to $u_{c 0}^{\Sigma}$, and the outer loop control target $\beta$ for the DC-axis can be selected as $P_{a c}$ or $u_{d c}$. A similar internal energy control for HB-MMC can be found in [21], which is proved to be effective in suppressing the oscillation between the DC bus capacitor and the sub-module capacitor.

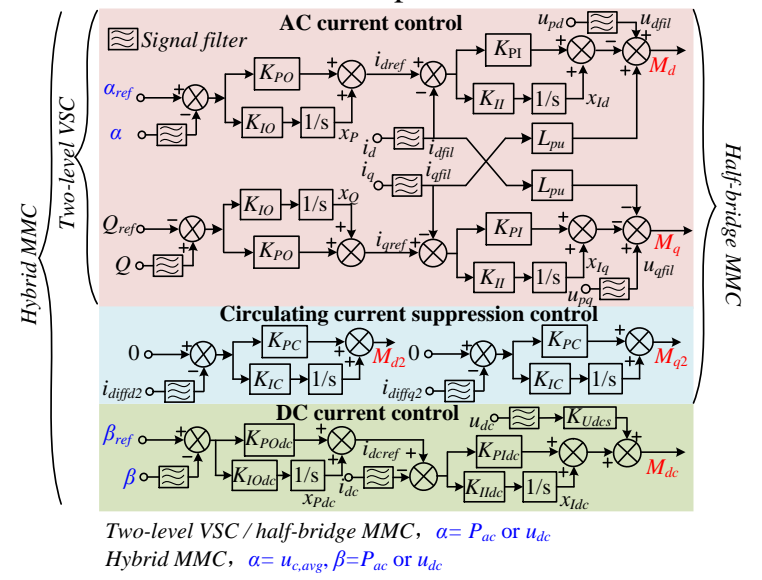

Fig. 4. Current controller structure for three types of VSCs.

TABLE I

COMPARISON OF THE SMALL-SIGNAL MODELS FOR VSCS 
The control system is modeled under the $d q$ rotating frame, which is aligned with the measured PCC voltage of the PLL. The relation between the $x y$ frame and the $d q$ frame can be derived based on the PLL dynamics. The dynamic model for the control system can be directly obtained based on block diagrams of the controller, which is not presented in detail in this paper. The dynamic models for PLL and signal filters can be addressed in [19]. By combining the dynamic models of different sub-systems, the whole small-signal model can be obtained in a modular way. The total orders for the closed-loop models of single TL-VSC, HB-MMC and HY-MMC are respectively 21,34 and 40 in this paper, as shown in TABLE I.

\section{IMPEDANCE ANALYSIS}

In this section, the $d q$ impedance models for TL-VSC, HBMMC and HY-MMC are derived and compared based on the small-signal model. The converters operate under the constant DC voltage and reactive power control mode. The output reactive power is set to be $10 \%$ of the rated capacity. The SCR of the connected $\mathrm{AC}$ system is set to be 2, which can be classified as the weak AC system. The parameters for the VSCs and $\mathrm{AC}$ system can be found in the Appendix. The parameters of the PLL, ACC and signal filters are kept the same for three VSCs, which can be also found in the Appendix. The timedelays of the sampled electric signals are simplified and represented by the second-order low-pass filters, as derived in the Appendix.

Limited by space, the derivations of the AC impedance models are provided in the Appendix, and the detailed derivation process can be referred to [11]-[13]. The impact of the DC-side network is neglected [10] [12] [28] by setting the small-signal expressions for the DC current or DC active power to be zeros. The $d q$ impedances of VSCs and the AC system are denoted as:

$$
\begin{gathered}
\mathbf{Z}_{\mathbf{c o n}}=\left[\begin{array}{ll}
Z_{\text {condd }} & Z_{\text {condq }} \\
Z_{\text {conqd }} & Z_{\text {conqq }}
\end{array}\right] \\
\mathbf{Z}_{\mathbf{g}}=\left[\begin{array}{cc}
Z_{\text {gdd }} & Z_{\text {gdq }} \\
Z_{\text {gqd }} & Z_{\text {gqq }}
\end{array}\right]=\left[\begin{array}{cc}
R_{s}+s L_{s} & -\omega_{0} L_{s} \\
\omega_{0} L_{s} & R_{s}+s L_{s}
\end{array}\right]
\end{gathered}
$$

\section{A. Impedance analysis based on bode plots}

The AC active power is gradually increased, and $\boldsymbol{Z}_{c o n}$ is calculated iteratively. By checking the phase differences of the $d q$ components of $\boldsymbol{Z}_{c o n}$ and $\boldsymbol{Z}_{g}$ where they have points of intersection in the magnitude response, the stability can be judged [15]. If the magnitude of $\boldsymbol{Z}_{g}$ is going to be larger than $Z_{c o n}$ and the phase difference is larger than $180^{\circ}$, the system is unstable.

For the inverter status, the values of $P_{a c}$ for TL-VSC, HBMMC and HY-MMC are all set to be $60 \%$ of the rated capacity. The frequency responses of $d q \mathrm{AC}$ impedances within $0.1 \mathrm{~Hz} \sim 1 \mathrm{kHz}$ are depicted in Fig. 5 (a). The negative resistance zones are shadowed. It can be seen that the frequency responses of $Z_{\text {conqq }}$ for the VSCs are almost identical, and the frequency responses of $Z_{\text {condd }}$ are similar. The $Z_{\text {condd }}$ is close to a pure positive resistance within low-frequency range $(<5 \mathrm{~Hz})$. It becomes a negative resistance within medium-frequency range $(<100 \mathrm{~Hz})$, and finally approaches a pure inductance as the frequency increases above $1 \mathrm{kHz}$. While $Z_{\text {conqq }}$ contains a negative resistance within about $200 \mathrm{~Hz}$, and becomes a positive resistance afterward.

Though there exist points of intersection between the magnitude curves of $Z_{\text {gdd }}$ and $Z_{\text {condd }}$, the corresponding phase differences are all within $180^{\circ}$. Instead, the instability is identified according to $Z_{q q}$, as depicted in Fig. 6 (a). For TLVSC, $Z_{g q q}$ becomes larger than $Z_{\text {conqq }}$ from $188.3 \mathrm{~Hz}$. The phases of $Z_{g q q}$ and $Z_{\text {conqq }}$ at $188.3 \mathrm{~Hz}$ are separately $88.84^{\circ}$ and $-95.87^{\circ}$, making up a phase difference of $184.7^{\circ}$, which indicates the system is unstable. For HB-MMC, $Z_{g q q}$ becomes larger than $Z_{\text {conqq }}$ from $187.7 \mathrm{~Hz}$, with a corresponding phase difference of $184.2^{\circ}$. For HY-MMC, $Z_{g q q}$ becomes larger than $Z_{\text {conqq }}$ from $187.6 \mathrm{~Hz}$, with a corresponding phase difference of $183.8^{\circ}$.
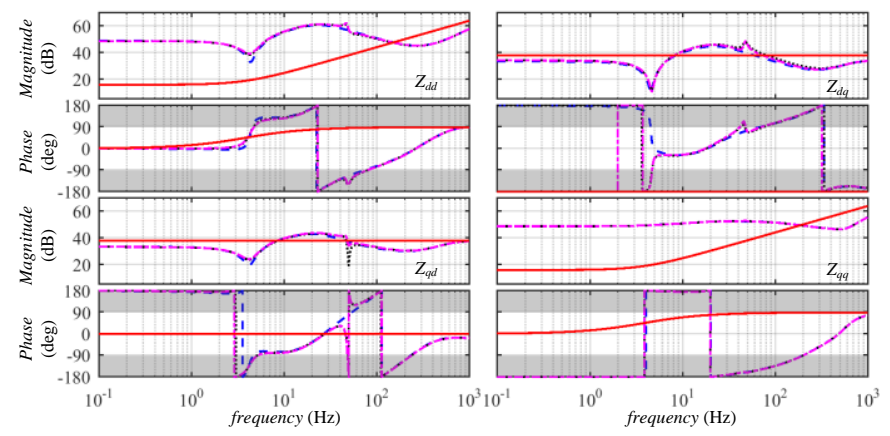

(a) frequency response of AC impedance under inverter operation

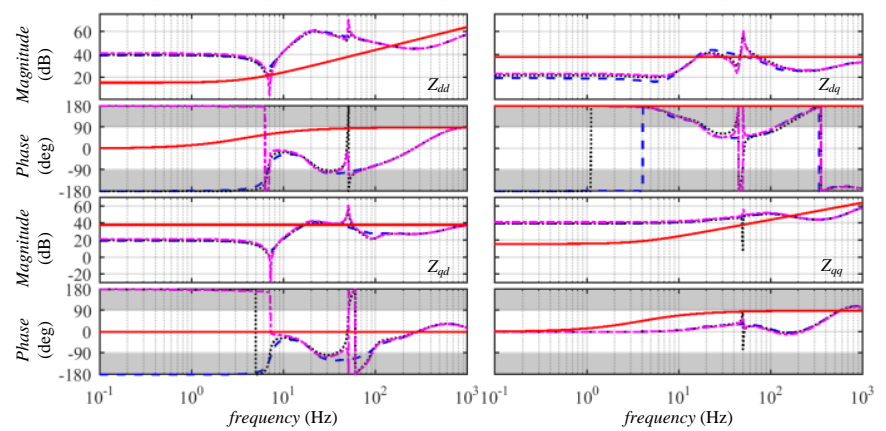

(b) frequency response of $\mathrm{AC}$ impedance under rectifier operation - - TL-VSC …… HB-MMC -.-. HY-MMC — AC Grid negative resistance zone Fig. 5. Comparison of AC impedances.

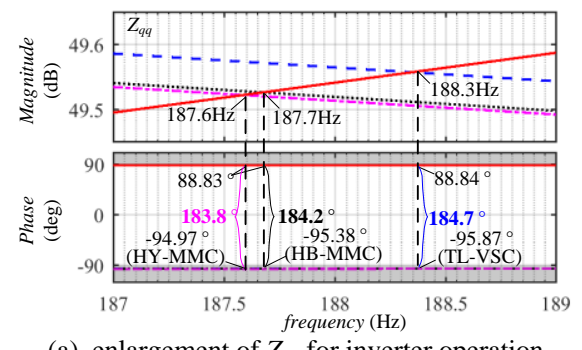

(a) enlargement of $Z_{q q}$ for inverter operation 


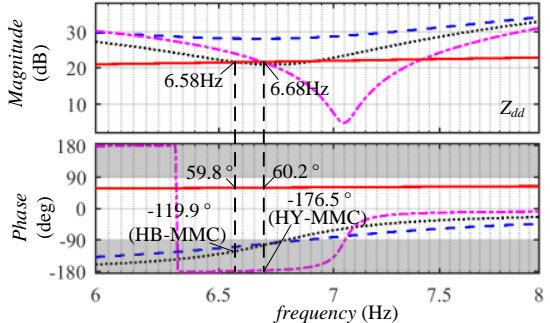

(b) enlargement of $Z_{d d}$ for rectifier operation

- - TL-VSC ........ HB-MMC -.-. HY-MMC — AC Grid negative resistance zone Fig. 6. Stability assessment.

For the rectifier status, $P_{a c}$ of TL-VSC, HB-MMC and HYMMC are all set to be $100 \%$ of the rated capacity. The frequency responses of $d q \mathrm{AC}$ impedance are depicted in Fig. 5 (b). $Z_{\text {condd }}$ is close to a pure negative resistance within lowfrequency range $(<7 \mathrm{~Hz})$. It becomes a positive resistance afterward, and finally approaches a pure inductance as the frequency increases above $1 \mathrm{kHz}$. $Z_{\text {conqq }}$ contains a positive resistance within $500 \mathrm{~Hz}$.

There also exist points of intersection between the magnitudes of $Z_{g q q}$ and $Z_{\text {conqq }}$, but the corresponding phase differences are all within $180^{\circ}$. Instead, the instability is identified according to $Z_{d d}$, as shown in Fig. 6 (b). TL-VSC is stable since $Z_{\text {gdd }}$ keeps smaller than $Z_{\text {condd }}$. For HB-MMC, the magnitude curves of $Z_{\text {condd }}$ and $Z_{g d d}$ cross at $6.58 \mathrm{~Hz}$, and $Z_{\text {gdd }}$ becomes bigger than $Z_{\text {condd }}$ afterward. But the phase difference at $6.58 \mathrm{~Hz}$ is within $180^{\circ}$, indicating a stable system. For HYMMC, the magnitude curves cross at $6.68 \mathrm{~Hz}$, and the corresponding phase difference is above $180^{\circ}$, indicating an unstable system.

\section{B. Impedance analysis based on generalized Nyquist criterion}

The stability can also be predicted by applying the generalized Nyquist criterion (GNC) to the impedance ratio $\mathbf{Z} \mathbf{g} / \mathbf{Z}_{\text {con. }}$. The eigenvalue loci of $\mathbf{Z}_{\mathrm{g}} / \mathbf{Z}_{\text {con }}$ for three VSCs are depicted in Fig. 7. The values of $P_{a c}$ under each operation state are set to be the same as those in Fig. 5. The critical point $(-1,0)$ is marked as ' + '. The two different eigenvalue loci are indicated by different arrows.

According to Fig. 7 (a), (c) and (e), the eigenvalue loci of the impedance ratio for three VSCs all encircle the point $(-1,0)$ clockwise, indicating instability under the inverter status.

In Fig. 7 (b) and (d), the eigenvalue loci of TL-VSC and HBMMC do not encircle $(-1,0)$, indicating stable systems. While in Fig. 7 (e), the eigenvalue loci of HY-MMC encircle $(-1,0)$ clockwise, indicating instability.

The GNC analysis results are consistent with the bode-plotbased impedance analysis regarding stability judgment. In

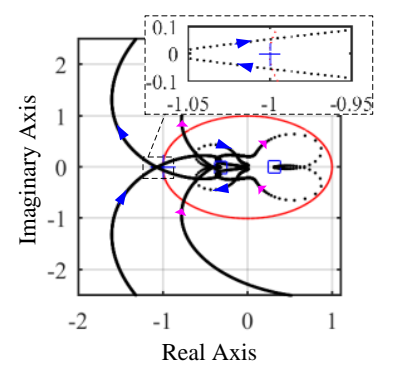

(a) inverted TL-VSC

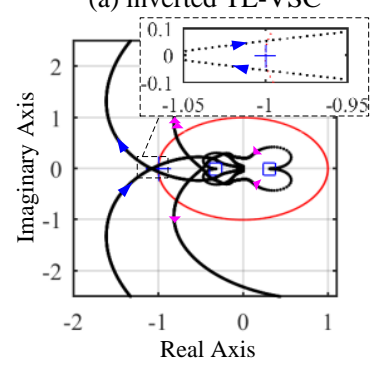

(c) inverted HB-MMC

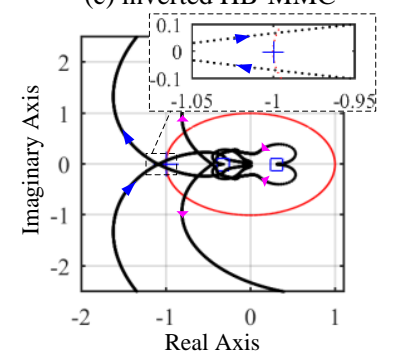

(e) inverted HY-MMC

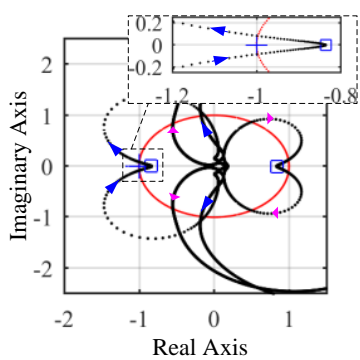

(b) rectified TL-VSC

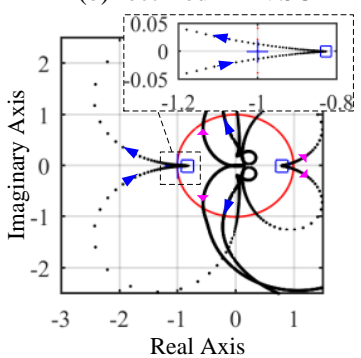

(d) rectified HB-MMC

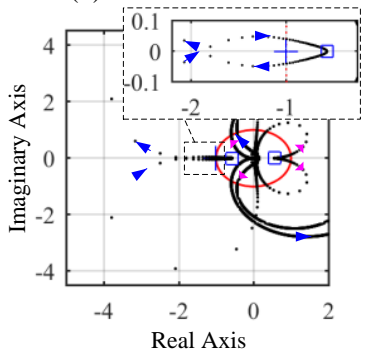

(f) rectified HY-MMC
Fig. 7. Nyquist diagrams of eigenvalue loci of $\mathbf{Z}_{\mathrm{g}} / \mathbf{Z}_{\text {con }}$.

comparison, the bode-plot-based analysis demonstrates more intuitive results, given that the oscillation frequencies and phase margins can be obtained from the magnitude-frequency and phase-frequency response curves straightforwardly.

\section{MOdAl ANALYSIS}

A two-terminal test system is employed in this section, as shown in Fig. 8. The master converter regulating the DC voltage and its reactive power is connected to the weak AC system, named as VSC1. The other terminal (VSC2) is the slave converter that controls its $\mathrm{PCC}$ active power and reactive power. The connected AC system 2 is with SCR of 20 . The capacitance and inductance of TL-VSCs and HB/HY-MMCs satisfy:

$$
\begin{gathered}
C_{d c}=6 C_{a r m} \\
\left(L_{T}+L_{a c} K_{T}^{2}\right)_{T L-V S C}=\left(L_{T}+0.5 L_{a r m} K_{T}^{2}\right)_{H B / H Y-M M C}
\end{gathered}
$$

\section{A. Root locus analysis}

Let the master converter respectively operate as an inverter or rectifier, and gradually increase the active power. The root loci for three topologies are depicted in Fig. 9 (a)-(c). As shown in the left column, with the rise of the inverted power into the weak AC system, all three test systems become unstable. Despite the different numbers and distribution of the eigenvalues, the oscillation frequencies of the unstable modes under the critical active power are respectively close to each other. The eigenvalue analysis complies with frequency- 
domain analysis in the previous section.

As shown in the right column of Fig. 9, with the rise of the rectified power from the weak AC system, all three test systems become unstable. The critical values of the active power and the corresponding oscillation frequencies of the unstable modes are noted in the figures, which are different from each other. However, the oscillation frequencies have the same order of magnitudes, which are all several $\mathrm{Hz}$.

\section{B. Participation factor analysis of state variables}

$\mathrm{PF}$ analysis is usually conducted to distinguish the most involved state variables regarding certain unstable modes. The $\mathrm{PF}$ of $k$ th state variable towards $i$ th eigenvalue is calculated [22]:

$$
p_{k i}=v_{k i} u_{k i} / \boldsymbol{v}_{i}^{T} \boldsymbol{u}_{i}
$$

where $\boldsymbol{v}_{i}$ and $\boldsymbol{u}_{i}$ are respectively the left and right eigenvectors for the $i$ th eigenvalue, and $v_{k i}$ and $u_{k i}$ are respectively the elements on the $k$ th row and $i$ th column of $\boldsymbol{v}_{i}$ and $\boldsymbol{u}_{i}$.

The values of the inverted and rectified active power are all set to be $60 \%$, and corresponding PF analysis results for the unstable modes are respectively displayed in Fig. 10 (a)-(c). The total orders of the test two-terminal systems are respectively 49,75 and 87 , including the models for $\mathrm{AC}$ currents and DC networks. The corresponding state variables for different sub-systems are labeled along the horizon axis in Fig. 10. Based on the distribution of the PF, similarity and difference can be obtained.

First of all, the mainly participated state variables under the inverter and rectifier status are different from each other, which holds for all the three topologies. Specifically, the unstable mode under the inverter status is primarily concerned with the reactive component of the AC current or measured PCC voltage, which are noted as $i_{y}$ and $u_{q f i l}$. In contrast, the unstable mode under the rectifier status is mainly related to the state variable of the outer loop for $d$-axis $x_{P}$ and the DC component of the capacitor voltage $\left(u_{d c}\right.$ or $\left.u_{c 0}^{\Sigma}\right)$. Notice that $x_{P}$ in the master VSCs is closely linked to the integration of $u_{d c}$ or $u_{c 0}^{\Sigma}$, so it can be inferred that the instability is closely concerned with $u_{d c}$ or $u_{c 0}^{\Sigma}$.

Secondly, the unstable mode under the inverter status can be classified as a local oscillation mode for all three VSCs: the related state variables only belong to the converter connected to the weak AC system. In comparison, the unstable mode under the rectifier status is an interactive oscillation mode for TLVSC and HB-MMC: the unstable mode is related to the $u_{d c}$ or $u_{c 0}^{\Sigma}$ of the other terminal connected to the strong AC system. This conclusion is consistent with [23], which reveals that the coupling effect between the AC and DC side dynamics cannot be ignored for studying low-frequency resonance of VSC. However, for HY-MMC, the unstable mode under the rectifier status is still a local oscillation mode, indicating less affected by the coupling effect.

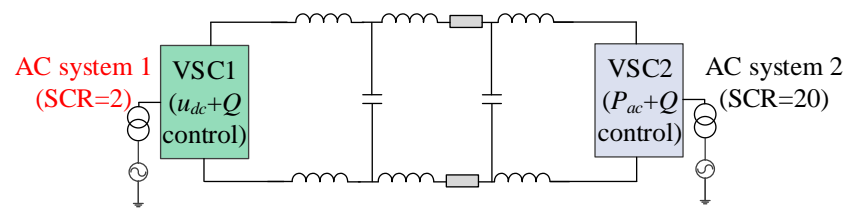

Fig. 8. Topology of the tested two-terminal system.
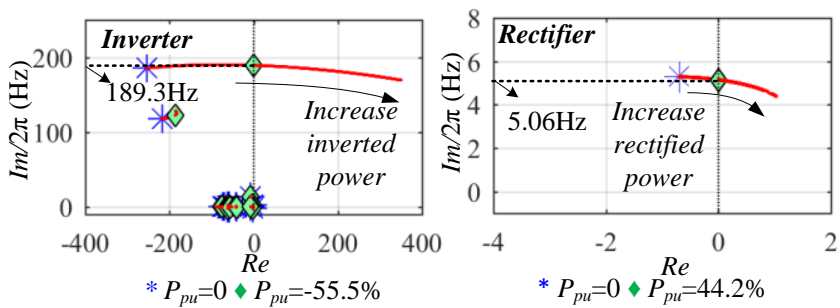

(a) TL-VSC
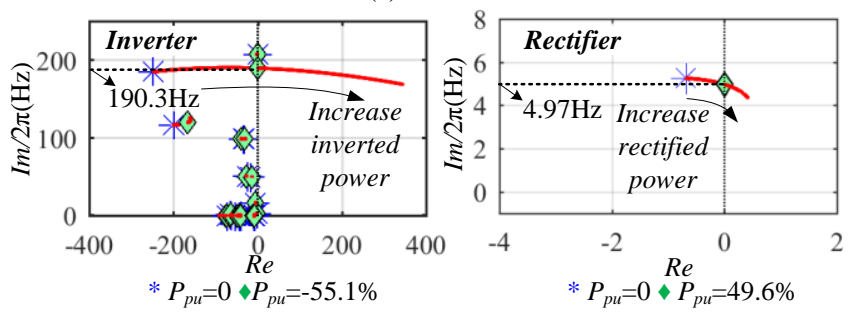

(b) HB-MMC

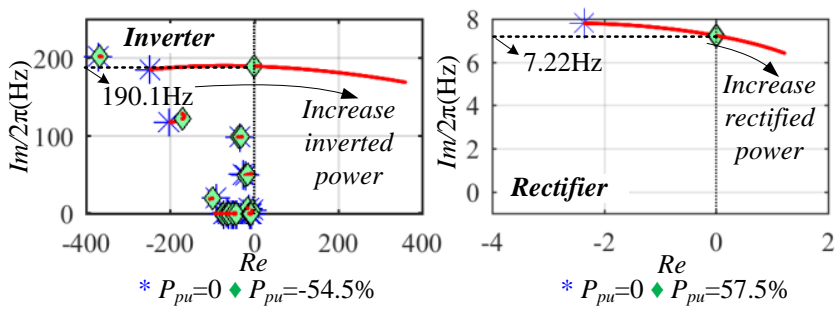

(c) HY-MMC

Fig. 9. Root locus analysis with increasing the active power.

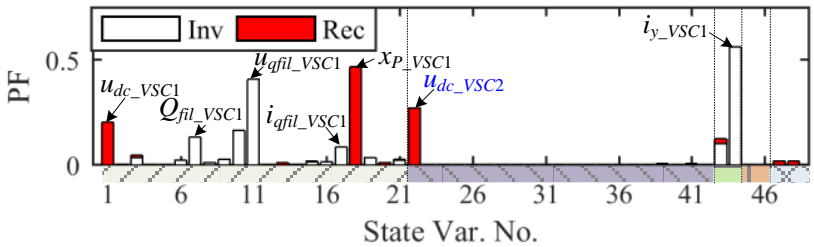

(a) TL-VSC

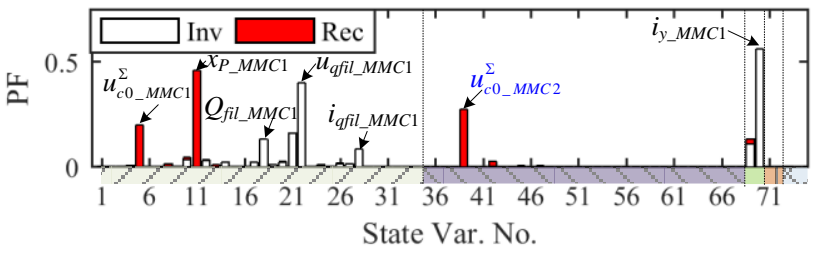

(b) HB-MMC

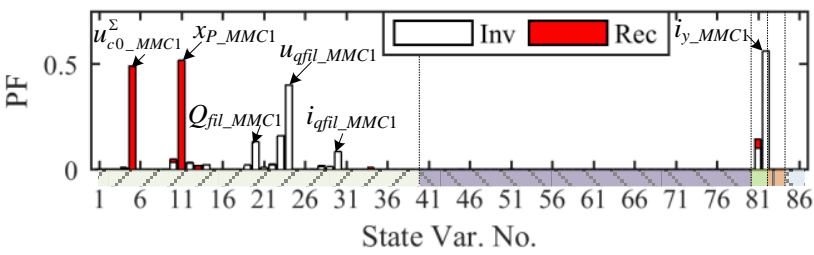

(c) HY-MMC

VSC1 VSC2 $=A C 1 \amalg A C 2 \times D C$ Netwrok

Fig. 10. Participation factor analysis under rectifier and inverter status. 


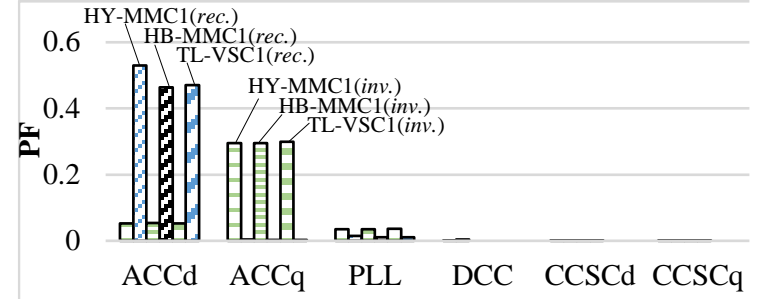

Fig. 11. Participation factor of different control loops versus the critical unstable modes under rectifier and inverter status.

\section{C.PF analysis of different control loops}

In order to figure out the participation of the control loops, such as the ACC $d$-axis (ACCd), ACC $q$-axis (ACCq), PLL, CCSC or DCC, we aggregate the PFs of state variables related to the corresponding control loop, including the inputs and the integration units. Taking the example of ACCd, the control output is $M_{d}$. According to Fig. 4, the small-signal model of $M_{d}$ can be expressed as:

$$
\begin{gathered}
\Delta M_{d}=\Delta u_{d f i l}+L_{p u} \Delta i_{q f i l}-\Delta x_{I d}+ \\
K_{P I}\left(\Delta i_{d f i l}-\Delta x_{P}+K_{P O} \Delta u_{d c f i l}\right)
\end{gathered}
$$

Then, the PF of ACCd can be equivalently calculated as:

$$
p_{A C C d}=p_{u_{d f f l}}+L_{p u} p_{i_{q f f l}}-p_{x_{l d}}+K_{P I}\left(p_{i_{d f f l}}-p_{x_{P}}+K_{P O} p_{u_{d f f l}}\right)
$$

where $p_{x}$ indicates the PF of $x$.

The calculation results are displayed in Fig. 11. The equivalent PF is not evident for PLL, DCC or CCSC, which means the controllability and observability of their outputs to the instability are little. Instead, to design the additional damping control for oscillation depression, the damping control input/output is better selected from/ injected into the ACCd under rectifier status or ACCq under inverter status.

\begin{tabular}{|c|c|c|c|c|}
\hline Status & Item & TL-VSC & "HB-MMC & "HY-MMC \\
\hline \multirow{5}{*}{ inverter } & critical $P_{a c}$ & $55.5 \%$ & $55.1 \%$ & $54.5 \%$ \\
\hline & osc. freq. & $189.3 \mathrm{~Hz}$ & $190.3 \mathrm{~Hz}$ & $190.1 \mathrm{~Hz}$ \\
\hline & state & $i_{y \_} V S C 1$, & $i_{y \_M M C 1}$, & $i_{y \_M M C 1}$, \\
\hline & variables & $u_{\text {qfil_VSC1}}$ & $u_{\text {qfil_MMC1 }}$ & $u_{\text {qfil_MMC1 }}$ \\
\hline & mode & Local & Local & Local \\
\hline \multirow{4}{*}{ rectifier } & critical $P_{a c}$ & $44.2 \%$ & $49.6 \%$ & $57.5 \%$ \\
\hline & osc. freq. & $5.06 \mathrm{~Hz}$ & $4.97 \mathrm{~Hz}$ & $7.22 \mathrm{~Hz}$ \\
\hline & $\begin{array}{c}\text { state } \\
\text { variables }\end{array}$ & $\begin{array}{l}x_{P_{-} V S C 1}, \\
u_{d c_{V} V S C 1}, \\
u_{d c_{-} V S C 2}\end{array}$ & $\begin{array}{l}x_{P_{-} M M C 1}, \\
u_{c 0 \_}^{\Sigma}, M M C 1 \\
u_{c 0_{-} M M C 2}^{\Sigma},\end{array}$ & $\begin{array}{l}x_{P \_M M C 1}, \\
u_{c 0 \_M M C 1}^{\Sigma}\end{array}$ \\
\hline & mode & Interactive & Interactive & Local \\
\hline
\end{tabular}

$$
\text { TABLE II }
$$

SUMMARY OF MODAL ANALYSIS RESULTS

D. Comparison with the AC impedance analysis

The frequency-domain impedance analysis in Section III shows that all the VSCs in inverter operation may suffer from an oscillation of hundreds-of $-\mathrm{Hz}$ at $60 \%$ active power transmission, which complies with the root locus analysis. In contrast, for the rectifier operation state, only HY-MMC may have an oscillation of several $\mathrm{Hz}$ at $100 \%$ active power transmission. The rectified TL-VSC and HB-MMC remain stable even at $100 \%$ active power transmission, which is not consistent with the modal analysis. The potential reason for this deviation on stability judgment is that the AC impedance model neglects the impact of the DC network and other terminals. The coupling of AC and DC networks is suggested to be considered for analyzing such low-frequency oscillation under rectifier operation [23]. Since the DC-side variables are closely related to the instability under rectifier operation, the neglect of the DC dynamics may cause an inaccurate estimation of the instability.

While for HY-MMC, the converter internal energy, as well as the DC current, is explicitly and independently regulated in different control loops, leading to more decoupled dynamics between AC and DC sides. Hence, the AC impedance analysis for HY-MMC is able to effectively predict the instability under both inverter and rectifier operation states.

\section{E. Summary}

The numbers of eigenvalues for the three types of VSCs are different from each other. Despite that MMCs produce more eigenvalues, the critical eigenvalues that induce instability are similar. The summary of the modal analysis is displayed in TABLE II. It can be concluded that,

- The instability triggered by increasing the inverted active power for the three types of VSCs shows the same characteristics.

- The instability triggered by increasing the rectified active power for the TL-VSC and HB-MMC shows the same characteristics. The difference for HY-MMC is that the unstable mode is only related to the local converter.

\section{V.SIMULATION VERIFICATION OF INSTABILITY}

In this section, the electromagnetic models of the test system under three VSC topologies are established in PSCAD/EMTDC. The instability is simulated, and corresponding oscillation is analyzed. The waveforms of the PCC active power $\left(P_{a c p u}\right)$, the DC current $\left(i_{d c p u}\right)$, the reactive power $\left(Q_{p u}\right)$, the DC voltage $\left(u_{d c p u}\right)$, phase A current $\left(i_{a_{-} V S C 1}, i_{a_{-} V S C 2}, i_{a_{-} M M C 1}, i_{a_{-} M M C 2}\right)$ and the average capacitor voltage $\left(u_{c 0}^{\Sigma}\right)$ are depicted. Harmonic percent (Harm.\%) of the oscillation waveforms is analyzed.

\section{A. $T L-V S C$}

As shown in Fig. 12 (a), the inverted active power $P_{\text {acpu }}$ of the TL-VSC1 gradually increases to $58 \%$, and the oscillation is triggered, which is reflected in $Q_{p u}, u_{d c p u}$ and $i_{d c p u}$. Fourier analysis shows that the dominant oscillation frequency is $192.4 \mathrm{~Hz}$, which is close to the modal calculation result of $189.0 \mathrm{~Hz}$ at $P_{\text {acpu }}=-58 \%$. Meanwhile, according to the frequency coupling of the rotating $d q$ frame and static $A B C$ frame, $143 \mathrm{~Hz}$ and $193 \mathrm{~Hz}$ harmonics appear in the AC current of VSC1, and the AC current of VSC2 contains little harmonics, as shown in Fig. 12 (c).

The rectified active power of VSC1 gradually increases to $45 \%$, and the oscillations in $u_{d c p u}, i_{d c p u}$ and $P_{a c p u}$ finally diverge, while $Q_{p u}$ remains stable, as shown in Fig. 12 (b). Fourier analysis shows that the dominant oscillation frequency is $5.00 \mathrm{~Hz}$, which is consistent with the modal calculation result of $5.05 \mathrm{~Hz}$ at $P_{\text {acpu }}=45 \%$. Meanwhile, $45 \mathrm{~Hz}$ and $55 \mathrm{~Hz}$ harmonics are observed in the AC current of VSC1, and the AC current of 
VSC2 contains little harmonics.

It can be seen that under both scenarios, the oscillation appears in the DC voltage and DC current of the master TLVSC1, which means the oscillation can be propagated into the DC network and affects the other terminals. However, the oscillation cannot pass the other converters and influence the corresponding connected AC system.

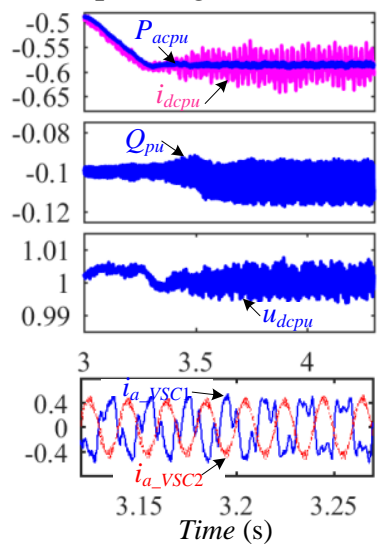

(a) simulation for the inverter

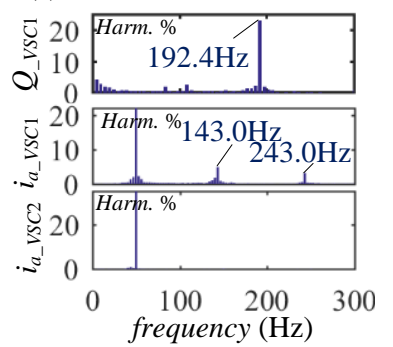

(c) harmonic analysis for the inverter

Fig. 12. Simulation results of TL-V

\section{B. $H B-M M C$}

As shown in Fig. 13 (a), the inverted active power of HBMMC1 gradually increases to 55\%. It is evident that diverging oscillation with high-frequency appears in $Q_{p u}, u_{d c p u}$ and $i_{d c p u}$, while mild oscillation is observed in both $u_{c 0_{-} M M C 1}^{\Sigma}$ and $u_{c 0 \_M M C 2}^{\Sigma}$. The dominant frequency of the oscillation is $208.7 \mathrm{~Hz}$, which is close to the modal calculation result of $190.3 \mathrm{~Hz}$. Meanwhile, corresponding harmonics can be observed in the $\mathrm{AC}$ current of HB-MMC1, but no harmonics appear in the AC current of HB-MMC2, as shown in Fig. 13 (c).

The rectified active power rises to 50\%, as shown in Fig. 13 (b), and low-frequency oscillation occurs in $u_{d c p u}, i_{d c p u}$, and $u_{c 0_{-} M M C 1}^{\Sigma}$, with the reactive power staying relatively stable. The dominant frequency of the oscillation is $4.91 \mathrm{~Hz}$, which is consistent with the modal calculation result of $4.97 \mathrm{~Hz}$. Corresponding harmonics appear in the AC current of HBMMC1 by frequency coupling. Moreover, $u_{c 0_{-} M M C 2}^{\Sigma}$ contains low-frequency oscillation, which accords with the PF analysis in Fig. 10 (b), but no harmonics emerge in the AC current of HB-MMC2.

It can be similarly concluded that the oscillation of HB-

MMC1 is propagated through the DC network and affects the other terminals. However, the oscillation is effectively absorbed and blocked by the sub-module capacitors of other MMCs, leaving the connected AC system unaffected.

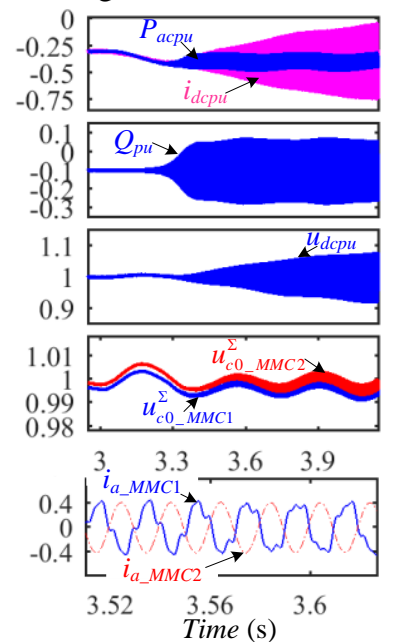

(a) simulation for the inverter

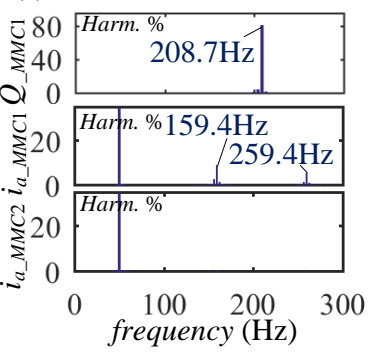

(c) harmonic analysis for the inverter Fig. 13. Simulation results of HB-MMCI

\section{C. $H Y-M M C$}

The instability under the inverter and rectifier status for the HY-MMC1 is simulated, as shown in Fig. 14 (a) and (b) respectively. Compared to the simulation results for the HBMMC1 in Fig. 13 (a) and (b), apparently, the DC current and DC voltage in Fig. 14 are nearly kept unaffected. As shown in Fig. 14 (b), the average capacitor voltage of MMC1 is oscillating, while that of MMC2 stays stable. Separate Fourier analysis to the oscillating reactive power and the average capacitor voltage reveals the dominant frequency components are respectively $186.3 \mathrm{~Hz}$ and $7.21 \mathrm{~Hz}$, which are consistent with the modal calculation results of $190.1 \mathrm{~Hz}$ and $7.22 \mathrm{~Hz}$. Meanwhile, corresponding harmonics appear in the AC current of HY-MMC1 due to frequency coupling, and the AC current of HY-MMC2 stays undistorted, as shown in Fig. 14 (c) and (d).

Compared to TL-VSC1 or HB-MMC1, for HY-MMC1, the oscillation cannot be propagated through the DC network. The oscillation or harmonics only appear on the AC-side, and are entirely absorbed and blocked by the master HY-MMC1, leaving the rest of the DC system unaffected. 


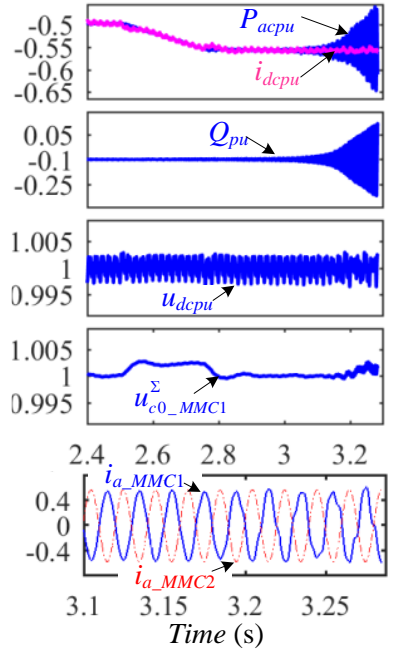

(a) simulation for the inverter

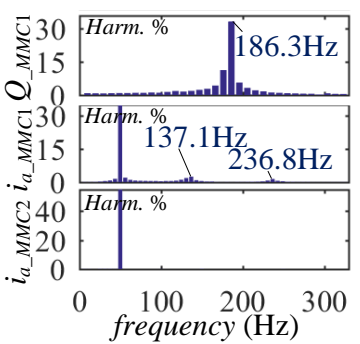

(c) harmonic analysis for the inverter

(d) harmonic analysis for the rectifier

\section{IMPROVEMENT OF STABILITY}

\section{A. Impact of the reactive power}

In practice, the unity power factor is normally required for the VSC-HVDC operation in order to make full use of the converter capacity. For weak AC grid integration, VSCs may be required to output reactive power to support the AC voltage [24][25]. The relationship between $u_{s}, u_{p}, P_{a c}$ and $Q$ can be obtained as [25]:

$$
u_{p}^{4}+\left(2 P_{a c} R_{s}-2 Q \omega L_{s}-u_{s}^{2}\right) u_{p}^{2}+\left(P_{a c}^{2}+Q^{2}\right)\left(\omega^{2} L_{s}^{2}+R_{s}^{2}\right)=0
$$

To guarantee real solutions of $u_{p}$ to (25), $P_{a c}$ and $Q$ of the operation point should satisfy:

$$
\Delta=\left(2 P_{a c} R_{s}-2 Q \omega L_{s}-u_{s}^{2}\right)^{2}-4\left(P_{a c}^{2}+Q^{2}\right)\left(\omega^{2} L_{s}^{2}+R_{s}^{2}\right) \geq 0
$$

The feasible operating points are calculated and gathered in Fig. 15, where the negative sign indicates capacitive reactive power from VSCs. The maximum apparent power is set to be $120 \%$ of the rated capacity. The magnitude of corresponding $u_{p}$ at each operation point is indicated by the color bar.

For a fixed reactive power value, the mathematically maximum active power can be obtained to ensure feasible operation points. The default reactive power for the VSCs in this paper is set to be $-10 \%$, then the calculated maximum active power is $118 \%$ for the inverter operation and $101 \%$ for the rectifier operation according to Fig. 15.

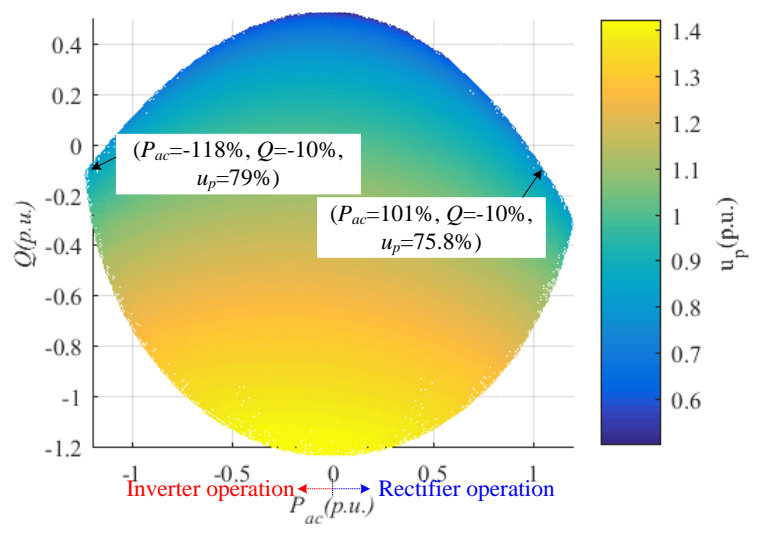

Fig. 15 Feasible operation zone considering the PCC voltage.

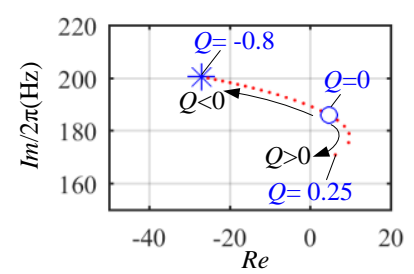

(a) inverter operation

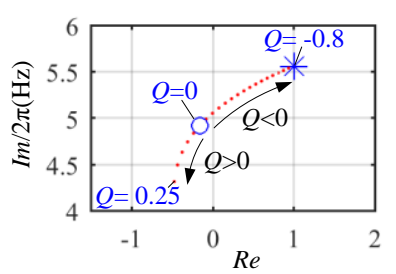

(b) rectifier operation
Fig. 16. Root locus analysis of varying the reactive power for TL-VSC.

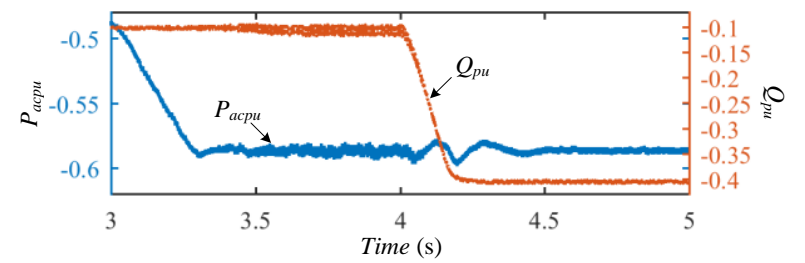

(a) inverter operation

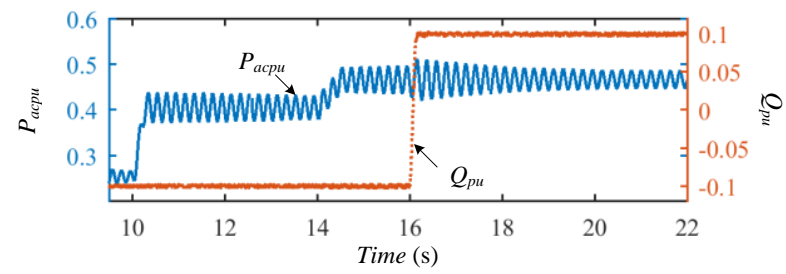

(b) rectifier operation

Fig. 17. Electromagnetic simulation of varying the reactive power.

Besides the above steady-state analysis, the impact of the reactive power on the small-signal stability is studied through the system root locus analysis. The calculation results only for TL-VSC is demonstrated. The results are similar for HB-MMC and HY-MMC. The values for the active power are selected as the critical values in Fig. 9 (a), i.e., $55.5 \%$ for the inverter operation and $44.2 \%$ for the rectifier operation.

According to Fig. 15, at such active power transmission, the feasible reactive power can be varied from $-80 \%$ to $25 \%$. Then the variation range of the reactive power for the root locus analysis is determined. As shown in Fig. 16 (a), under inverter operation, VSCs should output more reactive power to improve stability. In contrast, under rectifier operation, VSCs should reduce reactive power output, or even absorb more reactive power, as shown in Fig. 16 (b). The requirements of the reactive power for stability improvement are opposite under different operation states. 
The corresponding electromagnetic simulation results are depicted in Fig. 17. As shown in Fig. 17 (a), the high-frequency oscillation in the active and reactive power is effectively suppressed when the output reactive power is increased from $10 \%$ to $40 \%$. As shown in Fig. 17 (b), the amplitude of the lowfrequency oscillation in the active power is gradually decreasing as the reactive power is varied from $-10 \%$ to $10 \%$.

Compared with the simulation results in Fig. 19, adjusting the reactive power is not as quick and efficient as adjusting the control parameters in suppressing the oscillation. Moreover, with more output reactive power, the PCC voltage may be greatly increased, which may introduce the over-modulation problem for HB-MMC and HY-MMC, due to insufficient submodules to build the required $\mathrm{AC}$ voltage.

\section{B. Sensitivity study of control parameters}

Retuning of the control parameters can be useful for stability improvement. In [26]-[28], the speeding up of the AC voltage controller and the slowing down of PLL are suggested for the VSCs under DC voltage and AC voltage control mode. While in this paper, the VSCs are under a different control mode. Different results on retuning the control parameters are obtained, as demonstrated below.

The operating point are set as the critical states in Fig. 9 (a)(d). By separately varying the control parameters, the system root loci can be obtained, and the movement of the original unstable mode can be observed. The calculation results are depicted in Fig. 18.

As shown in Fig. 18 (a), for the TL-VSC inverter, by decreasing the default value of the proportional gain for PLL $K_{P p l l}$, the unstable mode moves to the left-half-plane, which indicates the improvement of stability. Similarly, decreasing the proportional and integration gain of ACC inner loop $\left(K_{P I}, K_{I I}\right)$ or outer loop $\left(K_{P O}, K_{I O}\right)$ can also help improve stability under inverter operation. The results for TL-VSC rectifier, as well as HB-MMC and HY-MMC, can be found in Fig. 18 (b)-(g).

The comparative results are demonstrated in TABLE III. It can be seen that:

(1) The operation state lays a great influence on PLL tuning. The control of PLL should be slowed down under inverter operation and accelerated under rectifier operation.

(2) Similarly to PLL tuning, the outer loop of ACC should be slowed down in $q$-axis under inverter operation and sped up in $d$-axis under rectifier operation.

Generally, under inverter status, the control gains for PLL and ACC, including $K_{P p l l}, K_{P I}, K_{I I}, K_{P O}$ and $K_{I O}$, need to be reduced to restore stability. In contrast, $K_{P p l l}$ and $K_{P O}$ need to be increased under rectifier status. Besides, the adjustment of DCC parameter for HY-MMC is only useful under rectifier status.

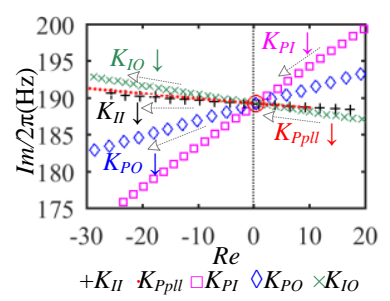

(a) TL-VSC inverter

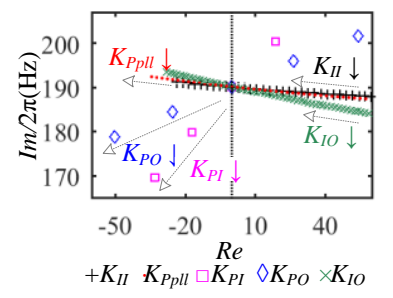

(c) HB-MMC inverter

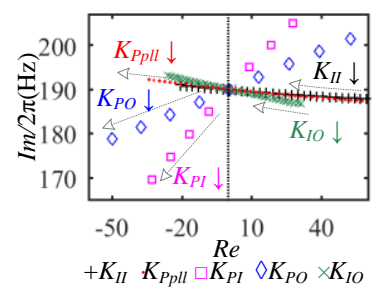

(e) HY-MMC inverter

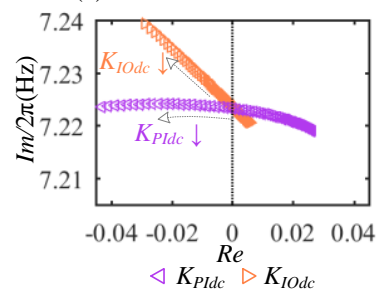

(g) HY-MMC rectifier

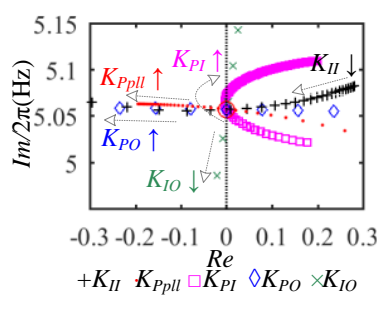

(b) TL-VSC rectifier

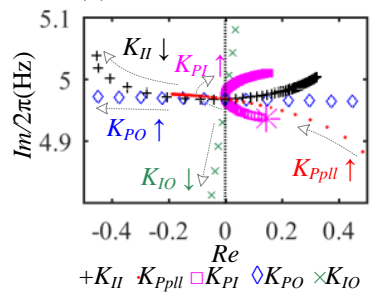

(d) HB-MMC rectifier
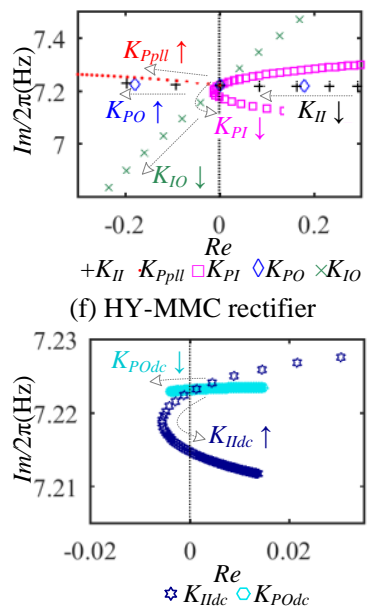

(h) HY-MMC rectifier (f) $\mathrm{HY}-\mathrm{MMC}$ rectifier

Fig. 18. Root locus of different control parameters.

TABLE III

SUGGESTED ADJUST DiRECTION OF CONTROL PARAMETERS FROM DEFAULTS

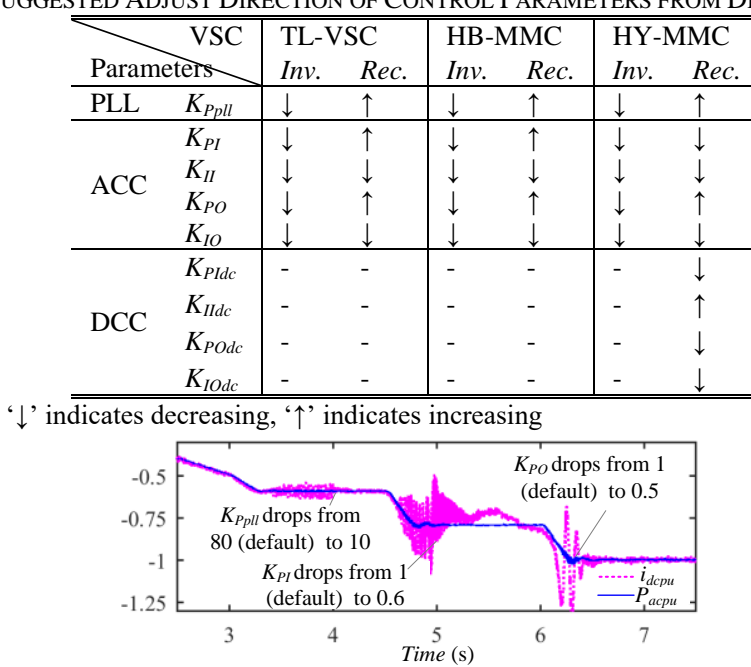

(a) inverter TL-VSC 


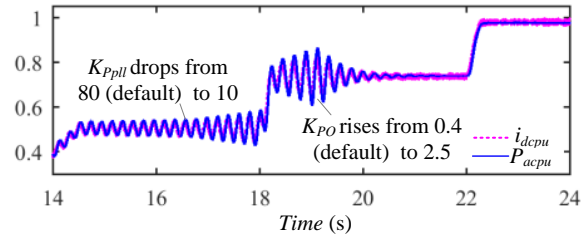

(b) rectifier TL-VSC

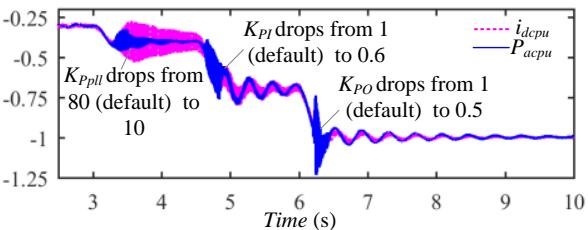

(c) inverter HB-MMC

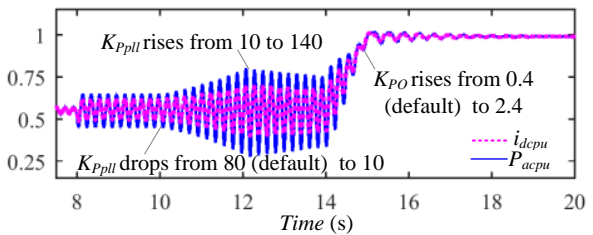

(d) rectifier HB-MMC

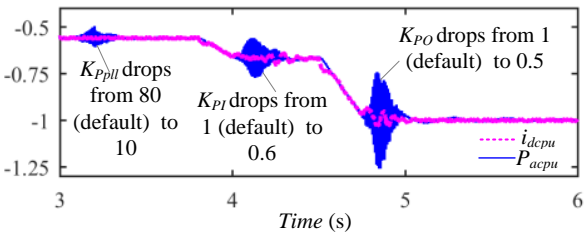

(e) inverter HY-MMC

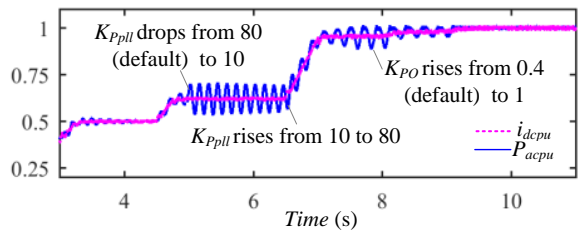

(f) rectifier HY-MMC

Fig. 19. Simulation results for validating effectiveness of parameter adjust.

\section{C.Simulation validation}

The effectiveness of adjusting control parameters is validated by simulation in PSCAD/EMTDC platform. The results are shown in Fig. 19.

As shown in Fig. 19 (a), (c) and (e), when high-frequency oscillation occurs in the inverted $\mathrm{AC}$ active power or DC current, $K_{P p l l}$ is reduced from default 80 to 10 , and the oscillation is effectively depressed. With the active power continuing rising, high-frequency oscillation emerges again, and $K_{P I}$ or $K_{P O}$ are successively reduced. The oscillation can be suppressed. The inverted active power finally achieves full capacity, as guaranteed by the calculation in Section VI. A.

As shown in Fig. 19 (b), (d) and (f), when low-frequency oscillation occurs in the rectified AC active power or DC current, if we still reduce $K_{P p l l}$ as we do under the inverter status, the oscillation is deteriorated with its amplitude increasing. After $K_{P O}$ is increased, the oscillation can be suppressed, and the rectified power successfully achieves full capacity, which is also confirmed by the calculation in Section VI. A.

\section{CONCLUSION}

This paper investigates the small-signal stability of TL-VSC, HB-MMC and HY-MMC under weak AC system integration by impedance analysis, root locus analysis, participation factor analysis and electromagnetic simulation.

Even though the small-signal model for the HB/HY-MMC is more complicated with considering the internal dynamics of the capacitor voltage and circulating current, $\mathrm{PF}$ analysis shows that except the DC capacitor voltage, other internal state variables of MMCs, such as the harmonics of the circulating current and total sub-module capacitor voltage, are hardly related to the instability.

The single-terminal impedance analysis for the three types of VSCs complies with the eigenvalue analysis results under the inverter operation state. However, it is unable to identify the instability under the rectifier operation state for TL-VSC and HB-MMC, which is due to the neglect of interaction with other DC terminals. While the unstable modes of HY-MMC are only related to local variables. It is due to the direct DC current control, which makes them decoupled from DC networks.

The two-terminal stability analysis results reveal that the stability characteristics of three types of VSCs under the inverter operation status are similar, including the frequencies of the oscillation, the mainly participating state variables and the effective parameter adjustment method. While under the rectifier operation status, the difference lies in the penetrability of the instability to other terminals. For TL-VSC and HB-MMC, the unstable modes are interactive, involving DC capacitor voltages from other converters. As for HY-MMC, the unstable mode is local, indicating the instability cannot affect other terminals through DC networks.

\section{APPENDIX}

A. Relations of time-delays and second-order low-pass filters The time-delay $\tau$ can be expressed as:

$$
D_{\text {delay }}(s)=e^{-\tau s}
$$

Applying Taylor series expansion to (A1) yields:

$$
D_{\text {delay }}(s)=\frac{1}{1+\tau s+\frac{\tau^{2} s^{2}}{2 !}+\frac{\tau^{3} s^{3}}{3 !}+\cdots}
$$

When $\tau$ is really small, we can neglect the higher orders, for example, the third order and above, then (A2) is simplified as:

$$
D_{\text {delay }}(s) \approx \frac{1}{1+\tau s+\frac{\tau^{2} s^{2}}{2 !}}
$$

(A3) is exactly the transfer function of a second-order lowpass filter with the damping ratio of 0.707 and the cutting-off frequency $f_{c}=\sqrt{2} / 2 \pi \tau$.

B. AC impedance of the TL-VSC

The small-signal model of $i$ : 


$$
\begin{aligned}
{\left[\begin{array}{c}
\Delta u_{p x} \\
\Delta u_{p y}
\end{array}\right]_{\Delta \mathbf{u}_{\mathrm{pxy}}}=} & \frac{K_{T} u_{d c 0}}{2}\left[\begin{array}{c}
\Delta M_{x} \\
\Delta M_{y}
\end{array}\right]_{\Delta \mathrm{M}_{\mathrm{xy}}}+\frac{K_{T}}{2}\left[\begin{array}{c}
M_{x 0} \\
M_{y 0}
\end{array}\right]_{d c}+ \\
& \frac{1}{K_{T}}\left[\begin{array}{cc}
R_{T}+s L_{t o t} & -\omega_{0} L_{t o t} \\
\omega_{0} L_{t o t} & R_{T}+s L_{t o t}
\end{array}\right]_{\mathbf{G}_{\mathrm{i}}}\left[\begin{array}{c}
\Delta i_{x} \\
\Delta i_{y}
\end{array}\right]
\end{aligned}
$$

where $L_{t o t}=L_{T}+K_{T}^{2} L_{a c}$.

According to (A4), $\Delta \mathbf{i}_{\mathbf{x y}}=\left[\Delta i_{x}, \Delta i_{y}\right]^{\mathrm{T}}$ can be expressed as:

$$
\begin{aligned}
\Delta \mathbf{i}_{\mathbf{x y}} & =\left[-\mathbf{G}_{\mathbf{i}}^{-1} \frac{K_{T} u_{d c 0}}{2}\right]_{\mathbf{G}_{\mathrm{iMxy}}} \Delta \mathbf{M}_{\mathbf{x y}}+\left[-\mathbf{G}_{\mathbf{i}}^{-1}\right]_{\mathbf{G}_{\text {iuxy }}} \Delta \mathbf{u}_{\mathbf{p x y}} \\
& +\left[-\mathbf{G}_{\mathbf{i}}^{-1} \frac{K_{T}}{2}\left[\begin{array}{l}
M_{x 0} \\
M_{y 0}
\end{array}\right]\right]_{\mathbf{G}_{\text {iudc }}} \Delta u_{d c}
\end{aligned}
$$

Neglecting the dynamics of the DC current from the DC network, the small-signal mode of $u_{d c}$ is,

$$
C_{d c} s \Delta u_{d c}=\frac{3}{2 K_{T}} \Delta\left(\frac{u_{p x} i_{x}+u_{p y} i_{y}}{u_{d c}}\right)
$$

According to (A6), $\Delta u_{d c}$ can be further expressed as:

$$
\begin{aligned}
\Delta u_{d c}= & {\left[\frac{3\left[u_{p x 0} u_{p y 0}\right]}{2\left(u_{d c 0} C_{d c} s+i_{d c 0}\right)}\right]_{\mathbf{G}_{\text {udci }}} \Delta \mathbf{i}_{\mathbf{x y}}+} \\
& {\left[\frac{3\left[i_{x 0} i_{y 0}\right]}{2\left(u_{d c 0} C_{d c} s+i_{d c 0}\right)}\right]_{\mathbf{G}_{\text {udcu }}} \Delta \mathbf{u}_{\mathbf{p x y}} }
\end{aligned}
$$

The small-signal for the ACC output $\left[\Delta M_{d}, \Delta M_{q}\right]^{\mathrm{T}}$ :

$$
\begin{aligned}
{\left[\begin{array}{c}
\Delta M_{d} \\
\Delta M_{q}
\end{array}\right] } & =\left[\begin{array}{c}
\Delta u_{d f i l} \\
\Delta u_{q f i l}
\end{array}\right]+\left[\begin{array}{cc}
G_{i n} & L_{p u} \\
-L_{p u} & G_{i n}
\end{array}\right]\left[\begin{array}{l}
\Delta i_{d f i l} \\
\Delta i_{q f i l}
\end{array}\right]- \\
& {\left[\begin{array}{c}
0 \\
G_{\text {in }} G_{\text {out }}
\end{array}\right] \Delta Q_{\text {fil }}+\left[\begin{array}{c}
G_{\text {in }} G_{\text {out }} \\
0
\end{array}\right] \Delta u_{\text {dffil }} }
\end{aligned}
$$

where $G_{i n}=K_{P I}+K_{I I} / s, G_{\text {out }}=K_{P O}+K_{I O} / s$.

Consider the transfer functions of the signal filters, such as the AC voltage,

$$
\left[\begin{array}{l}
\Delta u_{d f i l} \\
\Delta u_{q f i l}
\end{array}\right]_{\Delta \mathbf{u}_{\mathrm{dqfil}}}=\left[\frac{1 / U_{\text {base }}}{1+\tau_{u} s+\tau_{u}^{2} s^{2} / 2 !}\right]_{D_{u}}\left[\begin{array}{l}
\Delta u_{p d} \\
\Delta u_{p q}
\end{array}\right]_{\Delta \mathbf{u}_{\mathrm{pdq}}}
$$

where $\tau_{u}=\sqrt{2} / 2 \pi f_{\text {uac }}, f_{\text {uac }}$ is the cutting-off frequency for the AC voltage sampling filter. $U_{\text {base }}$ is the base value for the AC voltage. Similarly, we can obtain:

$$
\begin{gathered}
{\left[\begin{array}{c}
\Delta i_{d f i l} \\
\Delta i_{q f i l}
\end{array}\right]_{\Delta \mathbf{i}_{\mathrm{dqfil}}}=\left[\frac{1 / I_{\text {base }}}{1+\tau_{i} s+\tau_{i}^{2} s^{2} / 2 !}\right]_{D_{i}}\left[\begin{array}{c}
\Delta i_{d} \\
\Delta i_{q}
\end{array}\right]_{\Delta \mathbf{i}_{\mathrm{dq}}}} \\
\Delta Q_{\text {fil }}=\left[\frac{1 / S_{\text {base }}}{1+\tau_{Q} s+\tau_{Q}^{2} s^{2} / 2 !}\right]_{D_{Q}} \Delta Q \\
\Delta u_{d c f i l}=\left[\frac{1 / U_{d c b a s e}}{1+\tau_{u d c} s+\tau_{u d c}^{2} s^{2} / 2 !}\right]_{D_{u d c}} \Delta u_{d c}
\end{gathered}
$$

Then $\Delta \mathbf{M}_{\mathbf{d q}}=\left[\Delta M_{d}, \Delta M_{q}\right]^{\mathrm{T}}$ can be further expressed as:

$$
\begin{gathered}
\Delta \mathbf{M}_{\mathbf{d q}}=\left[\left[\begin{array}{l}
1,0 \\
0,1
\end{array}\right] D_{u}\right]_{\mathbf{G}_{\text {Mdqudq }}} \Delta \mathbf{u}_{\mathbf{p d q}}+\left[\left[\begin{array}{c}
0 \\
-G_{\text {in }} G_{\text {out }}
\end{array}\right] D_{Q}\right]_{\mathbf{G}_{\text {MdqQ }}} \Delta Q \\
+\left[\left[\begin{array}{cc}
G_{i n} & L_{p u} \\
-L_{p u} & G_{i n}
\end{array}\right] D_{i}\right]_{\mathbf{G}_{\text {Mdqidq }}} \Delta \mathbf{i}_{\mathbf{d q}}+\left[\left[\begin{array}{c}
G_{i n} G_{\text {out }} \\
0
\end{array}\right] D_{u d c}\right]_{\mathbf{G}_{\text {Mdqudc }}} \Delta u_{d c}
\end{gathered}
$$

The small-signal model for $Q$ :

$$
\begin{gathered}
\Delta Q=\left(3\left[-i_{y 0}, i_{x 0}\right] / 2 K_{T}\right)_{\mathbf{G}_{\mathrm{Qu}}} \Delta \mathbf{u}_{\mathbf{p x y}}+ \\
\left(3\left[u_{p y 0},-u_{p x 0}\right] / 2 K_{T}\right)_{\mathbf{G}_{\mathrm{Qi}}} \Delta \mathbf{i}_{\mathbf{x y}}
\end{gathered}
$$

The small-signal model for PLL:

$$
\Delta \theta=\left(\left[-\frac{1}{s}\left(K_{\text {Ppll }}+K_{\text {Ipll }} / s\right)\right] D_{u}[0,1]\right)_{\mathbf{G}_{\mathbf{P L L u}}} \Delta \mathbf{u}_{\mathbf{p d q}}
$$

$\left[\Delta i_{d}, \Delta i_{q}\right]^{\mathrm{T}}$ can be expressed as:

$$
\begin{aligned}
{\left[\begin{array}{l}
\Delta i_{d} \\
\Delta i_{q}
\end{array}\right] } & =\left[\begin{array}{cc}
\cos \theta_{0} & \sin \theta_{0} \\
-\sin \theta_{0} & \cos \theta_{0}
\end{array}\right]_{\mathbf{C}_{\theta 1}}\left[\begin{array}{l}
\Delta i_{x} \\
\Delta i_{y}
\end{array}\right]+ \\
& {\left[\begin{array}{cc}
-\sin \theta_{0} & \cos \theta_{0} \\
-\cos \theta_{0} & -\sin \theta_{0}
\end{array}\right]_{\mathbf{C}_{\theta 2}}\left[\begin{array}{l}
i_{x 0} \\
i_{y 0}
\end{array}\right]_{\mathrm{i}_{x y 0}} \Delta \theta }
\end{aligned}
$$

Substitute (A15) into (A16), and $\left[\Delta i_{d}, \Delta i_{q}\right]^{\mathrm{T}}$ can be obtained:

$$
\Delta \mathbf{i}_{\mathrm{dq}}=\mathbf{C}_{\theta 1} \Delta \mathbf{i}_{\mathrm{xy}}+\left(\mathbf{C}_{\theta 2} \mathbf{i}_{\mathrm{xy} 0} \mathbf{G}_{\text {PLLu }}\right)_{\mathbf{G}_{\text {idqudq }}} \Delta \mathbf{u}_{\mathrm{pdq}}
$$

Similarly, $\left[\Delta u_{p d}, \Delta u_{p q}\right]^{\mathrm{T}}$ can be obtained as:

$$
\Delta \mathbf{u}_{\text {pdq }}=\left[\left(\mathbf{I}-\mathbf{C}_{\boldsymbol{\theta} 2} \mathbf{u}_{\text {pxy0 }} \mathbf{G}_{\text {PLLu }}\right)^{-1} \mathbf{C}_{\boldsymbol{\theta} 1}\right]_{\mathbf{G}_{\text {udquxy }}} \Delta \mathbf{u}_{\text {pxy }}
$$

$\left[\Delta M_{x}, \Delta M_{y}\right]^{\mathrm{T}}$ can be expressed as:

$$
\begin{aligned}
& {\left[\begin{array}{l}
\Delta M_{x} \\
\Delta M_{y}
\end{array}\right]_{\Delta \mathbf{M}_{\mathrm{xy}}}=\left[\begin{array}{cc}
\cos \theta_{0} & -\sin \theta_{0} \\
\sin \theta_{0} & \cos \theta_{0}
\end{array}\right]_{\mathbf{C}_{03}}\left[\begin{array}{l}
\Delta M_{d} \\
\Delta M_{q}
\end{array}\right]+} \\
& {\left[\begin{array}{c}
-\sin \theta_{0}-\cos \theta_{0} \\
\cos \theta_{0}-\sin \theta_{0}
\end{array}\right]_{\mathbf{C}_{\theta 4}}\left[\begin{array}{l}
M_{d 0} \\
M_{q 0}
\end{array}\right]_{\mathbf{M}_{\mathrm{dq} 0}} \Delta \theta}
\end{aligned}
$$

Substituting (A14), (A17) and (A18) into (A13) yields:

$$
\begin{gathered}
\Delta \mathbf{M}_{\mathrm{dq}}=\left[\left(\mathbf{G}_{\mathrm{Mdqudq}}+\mathbf{G}_{\text {Mdqidq }} \mathbf{G}_{\text {idqudq }}\right) \mathbf{G}_{\mathrm{udquxy}}+\right. \\
\left.\mathbf{G}_{\mathrm{MdqQ}} \mathbf{G}_{\mathrm{Qu}}\right]_{\mathbf{G}_{\mathrm{Mdquxy}}} \Delta \mathbf{u}_{\mathrm{pxy}}+\mathbf{G}_{\mathrm{Mdqudc}} \Delta u_{d c}+ \\
\left(\mathbf{G}_{\mathrm{MdqQ}} \mathbf{G}_{\mathrm{Qi}}+\mathbf{G}_{\text {Mdqidq }} \mathbf{C}_{\mathbf{\theta 1}}\right)_{\mathbf{G}_{\text {Mdqixy }} \Delta \mathbf{i}_{\mathrm{xy}}}
\end{gathered}
$$

Substituting (A7) and (A20) into (A19) yields:

$$
\begin{aligned}
& \Delta \mathbf{M}_{\mathrm{xy}}=\left[\mathbf{C}_{\mathbf{\theta 3}}\left(\mathbf{G}_{\text {Mdqixy }}+\mathbf{G}_{\text {Mdqudc }} \mathbf{G}_{\mathrm{udci}}\right)\right]_{\mathbf{G}_{\mathrm{Mxyi}}} \Delta \mathbf{i}_{\mathrm{xy}}+ \\
& {\left[\mathbf{C}_{\boldsymbol{\theta} 3}\left(\mathbf{G}_{\text {Mdquxy }}+\mathbf{G}_{\text {Mdqude }} \mathbf{G}_{\text {udcu }}\right)+\right.} \\
& \left.\mathbf{C}_{\mathbf{\theta 4}} \mathbf{M}_{\mathrm{dq} \mathbf{0}} \mathbf{G}_{\mathrm{PLLu}} \mathbf{G}_{\text {udquxy }}\right]_{\mathbf{G}_{\mathrm{Mxyu}}} \Delta \mathbf{u}_{\mathrm{pxy}}
\end{aligned}
$$

Substitute (A7) and (A21) into (A5), and we can obtain the $d q$ impedance as:

$$
\begin{aligned}
& \mathbf{Z}_{\text {con }}= K_{T}\left(\mathbf{G}_{\mathrm{iMxy}} \mathbf{G}_{\mathrm{Mxyu}}+\mathbf{G}_{\mathrm{iudc}} \mathbf{G}_{\mathrm{udcu}}+\mathbf{G}_{\mathrm{iuxy}}\right)^{-1} \cdot \\
&\left(\mathbf{I}-\mathbf{G}_{\mathrm{iMxy}} \mathbf{G}_{\mathrm{Mxyi}}-\mathbf{G}_{\mathrm{iudc}} \mathbf{G}_{\mathrm{udci}}\right)
\end{aligned}
$$

\section{C.AC impedance of the HB-MMC}

The small-signal model of $i$ :

$$
\left[\begin{array}{cc}
R_{t o t}+s L_{t o t} & -\omega_{0} L_{t o t} \\
\omega_{0} L_{t o t} & R_{t o t}+s L_{t o t}
\end{array}\right]_{\mathbf{G}_{\mathbf{i}}}\left[\begin{array}{c}
\Delta i_{x} \\
\Delta i_{y}
\end{array}\right]+\left[\begin{array}{c}
\Delta e_{v x} \\
\Delta e_{v y}
\end{array}\right]=\frac{1}{K_{T}}\left[\begin{array}{l}
\Delta u_{p x} \\
\Delta u_{p y}
\end{array}\right]
$$


where $L_{t o t}=L_{a r m} / 2+L_{T} / K_{T}^{2}, R_{t o t}=R_{a r m} / 2+R_{T} / K_{T}^{2}$.

Based on (9) in the context, $\Delta \mathbf{e}_{\mathrm{vxy}}=\left[\Delta e_{v x}, \Delta e_{v y}\right]^{\mathrm{T}}$ are obtained:

$$
\begin{aligned}
& \Delta \mathbf{e}_{\mathbf{v x y}}=\left[\begin{array}{cc}
\frac{u_{c x 0}^{\Sigma}}{4} & \frac{u_{c y 0}^{\Sigma}}{4} \\
\frac{-u_{c y 0}^{\Sigma}}{4} & \frac{u_{c x 0}^{\Sigma}}{4}
\end{array}\right]_{\mathbf{G}_{\text {evM2 }}}\left[\begin{array}{l}
\Delta M_{x 2} \\
\Delta M_{y 2}
\end{array}\right]_{\Delta \mathbf{M}_{\mathbf{x y} 2}}+ \\
& {\left[\begin{array}{cc}
\frac{2 u_{c 00}^{\Sigma}+u_{p x 20}^{\Sigma}}{4} & \frac{u_{c y 20}^{\Sigma}}{4} \\
\frac{u_{c y 20}^{\Sigma}}{4} & \frac{2 u_{c 00}^{\Sigma}-u_{c x 20}^{\Sigma}}{4}
\end{array}\right]_{\mathbf{G}_{\mathrm{evM}}}\left[\begin{array}{l}
\Delta M_{x} \\
\Delta M_{y}
\end{array}\right]_{\Delta \mathbf{M}_{\mathrm{xy}}}+} \\
& {\left[\begin{array}{cccc}
\frac{M_{x 0}}{4} & \frac{M_{y 0}}{4} \frac{M_{x 20}-2}{4} & \frac{M_{y 20}}{4} & \frac{M_{x 0}}{2} \\
\frac{-M_{y 0}}{4} \frac{M_{x 0}}{4} & \frac{M_{y 20}}{4} & -\frac{M_{x 20}+2}{4} \frac{M_{y 0}}{2}
\end{array}\right]_{\mathbf{G}_{\text {evuc }}} \Delta \mathbf{u}_{\mathbf{c}}^{\mathrm{\Sigma}}}
\end{aligned}
$$

The small-signal model of $\Delta \mathbf{u}_{\mathbf{c}}^{\Sigma}$ is [19]:

$$
\begin{aligned}
& \mathbf{G}_{\mathbf{u c}} \Delta \mathbf{u}_{\mathbf{c}}^{\Sigma}= \mathbf{G}_{\mathrm{uci} 0} \Delta i_{\text {diff } 0}+\mathbf{G}_{\mathrm{ucidiff}} \Delta \mathbf{i}_{\mathrm{diffxy} 2}+ \\
& \mathbf{G}_{\mathrm{ucM}} \Delta \mathbf{M}_{\mathrm{xy}}+\mathbf{G}_{\mathrm{ucM} 2} \Delta \mathbf{M}_{\mathrm{xy} 2}+\mathbf{G}_{\mathrm{uci}} \Delta \mathbf{i}_{\mathrm{xy}}
\end{aligned}
$$

where,

$$
\begin{aligned}
& \mathbf{G}_{\mathbf{u c}}=\left[\begin{array}{ccccc}
C_{a r m} s & -2 \omega C_{a r m} & 0 & 0 & 0 \\
2 \omega C_{a r m} & C_{a r m} s & 0 & 0 & 0 \\
0 & 0 & C_{a r m} s & -\omega C_{a r m} & 0 \\
0 & 0 & \omega C_{a r m} & C_{a r m} s & 0 \\
0 & 0 & 0 & 0 & C_{a r m} s
\end{array}\right] \\
& \mathbf{G}_{\text {uci0 }}=\frac{1}{2}\left[M_{x 20} M_{y 20} M_{x 0} M_{y 0}-1\right]^{\mathrm{T}} \\
& \mathbf{G}_{\text {ucidiff }}=\frac{1}{4}\left[\begin{array}{ccccc}
-2 & 0 & M_{x 0} & -M_{y 0} & M_{x 20} \\
0 & -2 & M_{y 0} & M_{x 0} & M_{y 20}
\end{array}\right]^{\mathrm{T}} \\
& \mathbf{G}_{\mathbf{u c M}}=\frac{1}{8}\left[\begin{array}{ccccc}
i_{x 0} & i_{y 0} & 4 i_{\text {diff } 00} & 0 & i_{x 0} \\
-i_{y 0} & i_{x 0} & 0 & 4 i_{\text {diff } 00} & i_{y 0}
\end{array}\right]^{\mathrm{T}} \\
& \mathbf{G}_{\mathbf{u c M 2}}=\frac{1}{8}\left[\begin{array}{ccccc}
4 i_{\text {diff } 00} & 0 & i_{x 0} & -i_{y 0} & 0 \\
0 & 4 i_{\text {diff } 00} & i_{y 0} & i_{x 0} & 0
\end{array}\right]^{\mathrm{T}}
\end{aligned}
$$

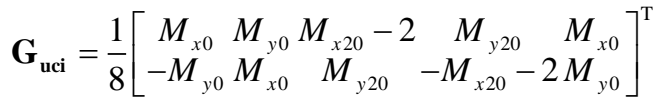

The small-signal model of $\Delta \mathbf{i}_{\text {diffxy } 2}=\left[\Delta i_{\text {diff } x 2}, \Delta i_{\text {diffy } 2}\right]^{\mathrm{T}}$ is [19]:

$\left[\begin{array}{cc}R_{\text {arm }}+L_{\text {arm }} s-2 \omega L_{\text {arm }} \\ 2 \omega L_{\text {arm }} & R_{\text {arm }}+L_{\text {arm }} s\end{array}\right]_{\mathbf{G}_{\text {idiff }}} \Delta \mathbf{i}_{\text {diffxy } 2}=$

$\left[\begin{array}{l}\frac{1}{2} 0-\frac{M_{x 0}}{4} \frac{M_{y 0}}{4}-\frac{M_{x 20}}{2} \\ 0 \frac{1}{2}-\frac{M_{y 0}}{4}-\frac{M_{x 0}}{4}-\frac{M_{y 20}}{2}\end{array}\right]_{\mathbf{G}_{\text {idiffuc }}} \Delta \mathbf{u}_{\mathbf{c}}^{\Sigma}+$

$\left[\begin{array}{cc}-\frac{u_{c x 0}^{\Sigma}}{4} & \frac{u_{c y 0}^{\Sigma}}{4} \\ -\frac{u_{c y 0}^{\Sigma}}{4}-\frac{u_{c x 0}^{\Sigma}}{4}\end{array}\right]_{\mathbf{G}_{\text {idiff }}} \Delta \mathbf{M}_{\mathbf{x y}}+\left[\begin{array}{cc}-\frac{u_{c 00}^{\Sigma}}{2} & 0 \\ 0 & -\frac{u_{c 00}^{\Sigma}}{2}\end{array}\right]_{\mathbf{G}_{\text {idiffM2 }}} \Delta \mathbf{M}_{\mathbf{x y 2}}$

The small-signal model of $\Delta i_{\text {diffo }}[19]$ :

$$
\begin{aligned}
& \left(s L_{a r m}+R_{a r m}\right)_{G_{i 0}} \Delta i_{d i f f 0}=\left(-\frac{1}{2}\right)_{G_{i 0 u d c}} \Delta u_{d c}+ \\
& {\left[\frac{-M_{x 20}}{4} \frac{-M_{y 20}}{4} \frac{-M_{x 0}}{4} \frac{-M_{y 0}}{4} \frac{1}{2}\right]_{\mathbf{G}_{\text {iouc }}} \Delta \mathbf{u}_{\mathbf{c}}^{\Sigma}+} \\
& {\left[\frac{-u_{c x 0}^{\Sigma}}{4} \frac{-u_{c y 0}^{\Sigma}}{4}\right]_{\mathbf{G}_{\text {i0 }}} \Delta \mathbf{M}_{\mathbf{x y}}+\left[\frac{-u_{c x 20}^{\Sigma}}{4} \frac{-u_{c y 20}^{\Sigma}}{4}\right]_{\mathbf{G}_{\text {i0 } 2}} \Delta \mathbf{M}_{\mathbf{x y 2} 2}}
\end{aligned}
$$

The small-signal models of PLL, $\Delta \mathbf{i}_{\mathbf{d q}}$ and $\Delta \mathbf{u}_{\text {pdq }}$ are the same as the TL-VSC. The small-signal model for ACC is the same as (A20), then $\Delta \mathbf{M}_{\mathbf{x y}}$ can be expressed as:

$$
\begin{gathered}
\Delta \mathbf{M}_{\mathbf{x y}}=\left(\mathbf{C}_{\mathbf{\theta 3}} \mathbf{G}_{\text {Mdqixy }}\right)_{\mathbf{G}_{\text {Mxyi }}} \Delta \mathbf{i}_{\mathbf{x y}}+\left(\mathbf{C}_{\boldsymbol{\theta} 3} \mathbf{G}_{\text {Mdqudc }}\right)_{\mathbf{G}_{\text {Mxyudc }}} \Delta u_{d c} \\
+\left(\mathbf{C}_{\boldsymbol{\theta} 3} \mathbf{G}_{\text {Mdquxy }}+\mathbf{C}_{\boldsymbol{\theta} 4} \mathbf{M}_{\mathrm{dq} \mathbf{0}} \mathbf{G}_{\text {PLLu }} \mathbf{G}_{\text {udquxy }}\right)_{\mathbf{G}_{\text {Mxyu }}} \Delta \mathbf{u}_{\text {pxy }}
\end{gathered}
$$

The small-signal model of CCSC:

$$
\left[\begin{array}{l}
\Delta M_{d 2} \\
\Delta M_{q 2}
\end{array}\right]_{\Delta \mathbf{M}_{\mathbf{d q} 2}}=\left(\left[\begin{array}{cc}
G_{C C} & 0 \\
0 & G_{C C}
\end{array}\right] D_{\text {idiff }}\right)_{\mathbf{G}_{\mathbf{M d q} 2}}\left[\begin{array}{l}
\Delta i_{\text {diffd } 2} \\
\Delta i_{\text {diff } q 2}
\end{array}\right]_{\Delta \mathbf{i}_{\text {diffd } 2}}
$$

where $G_{C C}=K_{P C}+K_{I C} / s$. $D_{\text {idiff }}$ is the transfer function of the signal filter for the circulating current.

$\Delta \mathbf{i}_{\text {diffdq } 2}$ can be expressed as:

$$
\Delta \mathbf{i}_{\text {diffdq } 2}=\left[\begin{array}{cc}
\cos 2 \theta_{0} & \sin 2 \theta_{0} \\
-\sin 2 \theta_{0} & \cos 2 \theta_{0}
\end{array}\right]_{\mathbf{C}_{05}} \Delta \mathbf{i}_{\text {diffxy } 2}
$$

$\left[\Delta M_{x 2}, \Delta M_{y 2}\right]^{\mathrm{T}}$ can be expressed as:

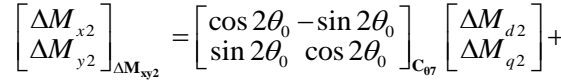

$$
\begin{aligned}
& {\left[\begin{array}{c}
-2 \sin 2 \theta_{0}-2 \cos 2 \theta_{0} \\
2 \cos 2 \theta_{0}-2 \sin 2 \theta_{0}
\end{array}\right]_{\mathrm{C}_{08}}\left[\begin{array}{l}
M_{d 20} \\
M_{q 20}
\end{array}\right]_{\mathrm{M}_{\mathrm{dq} 20}} \Delta \theta}
\end{aligned}
$$

Substitute (A29) and (A30) into (A31), and we can get:

$$
\begin{aligned}
& \Delta \mathbf{M}_{\mathrm{xy} 2}=\left(\mathbf{C}_{\theta 7} \mathbf{G}_{\mathrm{Mdq} 2} \mathbf{C}_{\theta 5}\right)_{\mathbf{G}_{\mathrm{Mxy2} 2}} \Delta \mathbf{i}_{\mathrm{diffxy} 2}+ \\
& \left(\mathbf{C}_{\theta 8} \mathbf{M}_{\mathrm{dq} 20} \mathbf{G}_{\text {PLLu }} \mathbf{G}_{\text {udquxy }}\right)_{\mathbf{G}_{\mathrm{Mx} 2 \mathrm{z}}} \Delta \mathbf{u}_{\mathrm{pxy}}
\end{aligned}
$$

By neglecting the deviation of DC active power, we get:

$$
0=3 u_{d c 0} \Delta i_{d i f f 0}+i_{d c 0} \Delta u_{d c}
$$

Then we get a group of algebra equations in complex field:

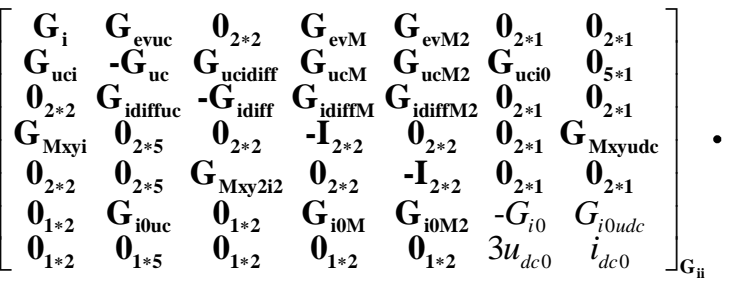

$$
\begin{aligned}
& {\left[\begin{array}{c}
\Delta \mathbf{i}_{\mathbf{x y}} \\
\Delta \mathbf{u}_{\mathbf{c}}^{\Sigma} \\
\Delta \mathbf{i}_{\text {diffxy2 }} \\
\Delta \mathbf{M}_{\mathbf{x y}} \\
\Delta \mathbf{M}_{\mathbf{x y 2}} \\
\Delta i_{d i f f 0} \\
\Delta u_{d c}
\end{array}\right]=\left[\begin{array}{c}
\mathbf{I}_{2 * 2} / K_{T} \\
\mathbf{0}_{5 * 2} \\
\mathbf{0}_{2 * 2} \\
-\mathbf{G}_{\mathbf{M x y u}} \\
-\mathbf{G}_{\mathbf{M x y 2 u}} \\
\mathbf{0}_{\mathbf{1} \mathbf{2}} \\
\mathbf{0}_{\mathbf{1} * \mathbf{2}}
\end{array}\right]_{\mathbf{G}_{\mathrm{uu}}} \Delta \mathbf{u}_{\mathbf{p x y}}}
\end{aligned}
$$

The direct analytical expression between $\Delta \mathbf{u}_{\mathbf{p x y}}$ and $\Delta \mathbf{i}_{\mathbf{x y}}$ is difficult to be obtained. However, we can iteratively solve (A34) in the numerical analysis software by frequency scanning. The 
first two rows of $\mathbf{G}_{\mathrm{ii}}^{-1} \mathbf{G}_{\text {uu }}$ are related to the AC admittance. Then we can get the AC impedance of the HB-MMC as:

$$
\mathbf{Z}_{\text {con }}=K_{T}\left(\mathbf{G}_{\mathrm{ii}}^{-1} \mathbf{G}_{\mathbf{u u}}([1,2],:)\right)^{-1}
$$

\section{D.AC impedance of the $H Y-M M C$}

The AC impedance modeling for HY-MMC is similar to that of HB-MMC, except that the small-signal model of DC current controller $\Delta M_{d c}$ should be considered. The small-signal model of AC current is same as (A23). The small-signal model of $\Delta \mathbf{e}_{\mathbf{v x y}}$ is modified as:

$$
\begin{aligned}
& \Delta \mathbf{e}_{\mathrm{vxy}}=\mathbf{G}_{\mathrm{evM}} \Delta \mathbf{M}_{\mathrm{xy}}+\mathbf{G}_{\mathrm{evM} 2} \Delta \mathbf{M}_{\mathrm{xy} 2}+\left[\frac{-u_{c x 0}^{\Sigma}}{2} \frac{-u_{c y 0}^{\Sigma}}{2}\right]_{\mathrm{G}_{\mathrm{e} \mathrm{Mdc}}}^{\mathrm{T}} \Delta M_{d c}+ \\
& {\left[\begin{array}{ccc}
\frac{M_{x 0}}{4} \frac{M_{y 0}}{4} \frac{M_{x 20}-2 M_{d c 0}}{4} & \frac{M_{y 20}}{4} & \frac{M_{x 0}}{2} \\
\frac{-M_{y 0}}{4} \frac{M_{x 0}}{4} & \frac{M_{y 20}}{4} & \frac{-M_{x 20}-2 M_{d c 0}}{4} \frac{M_{y 0}}{2}
\end{array}\right]_{\mathrm{G}_{\mathrm{ewc}}} \Delta \mathbf{u}_{\mathrm{c}}^{\Sigma}}
\end{aligned}
$$

Similarly, $\Delta M_{d c}$ shall appear in other small-signal models.

For the total capacitor voltage:

$$
\begin{aligned}
& \mathbf{G}_{\mathbf{u c}} \Delta \mathbf{u}_{\mathbf{c}}^{\Sigma}=\mathbf{G}_{\text {ucio }} \Delta i_{\text {diff } 0}+\mathbf{G}_{\text {ucidiff }} \Delta \mathbf{i}_{\text {diffxy } 2}+\mathbf{G}_{\text {ucMdc }} \Delta M_{d c} \\
& \mathbf{G}_{\mathrm{ucM}} \Delta \mathbf{M}_{\mathrm{xy}}+\mathbf{G}_{\mathrm{ucM} 2} \Delta \mathbf{M}_{\mathrm{xy} 2}+\mathbf{G}_{\mathrm{uci}} \Delta \mathbf{i}_{\mathrm{xy}} \\
& \mathbf{G}_{\text {uci } 0}=\frac{1}{2}\left[M_{x 20} M_{y 20} M_{x 0} M_{y 0}-M_{d c 0}\right]^{\mathrm{T}}
\end{aligned}
$$

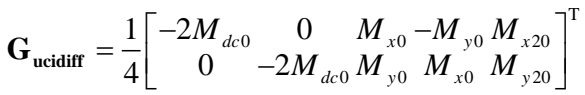

$$
\begin{aligned}
& \mathbf{G}_{\text {uci }}=\frac{1}{8}\left[\begin{array}{cccc}
M_{x 0} M_{y 0} M_{x 20}-2 M_{d c 0} & M_{y 20} & M_{x 0} \\
-M_{y 0} M_{x 0} & M_{y 20} & -M_{x 20}-2 M_{d c 0} M_{y 0}
\end{array}\right]^{\mathrm{T}} \\
& \mathbf{G}_{\text {ucMdc }}=\left[00 \frac{-i_{x 0}}{4} \frac{-i_{y 0}}{4} \frac{-i_{\text {diff } 00}}{2}\right]^{\mathrm{T}}
\end{aligned}
$$

For the second harmonic of the circulating current:

$$
\begin{aligned}
& \mathbf{G}_{\text {idiff }} \Delta \mathbf{i}_{\text {diffyy2 }}=\mathbf{G}_{\text {idiffm }} \Delta \mathbf{M}_{\mathrm{xy}}+\mathbf{G}_{\text {idiffM2 } 2} \Delta \mathbf{M}_{\mathrm{xy2}}+
\end{aligned}
$$

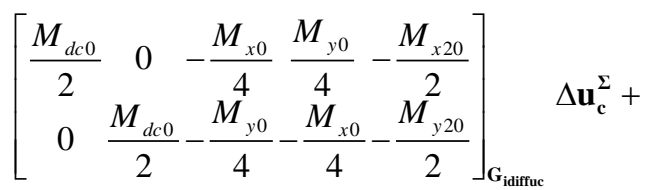

$$
\begin{aligned}
& {\left[\frac{u_{c x 20}^{\Sigma}}{2} \frac{u_{c y 20}^{\Sigma}}{2}\right]_{\mathbf{G}_{\text {idifrMde }}}^{\mathrm{T}} \Delta M_{d c}}
\end{aligned}
$$

For the DC component of the circulating current:

$$
\begin{aligned}
& G_{i 0} \Delta i_{d i f f 0}=G_{i 0 u d c} \Delta u_{d c}+\mathbf{G}_{\mathbf{i 0 M}} \Delta \mathbf{M}_{\mathbf{x y}}+\Delta \mathbf{G}_{\mathbf{i} \mathbf{0 M} 2} \mathbf{M}_{\mathbf{x y 2}}+ \\
& \quad\left[\frac{-M_{x 20}}{4} \frac{-M_{y 20}}{4} \frac{-M_{x 0}}{4} \frac{-M_{y 0}}{4} \frac{M_{d c 0}}{2}\right]_{\mathbf{G}_{\text {iouc }}} \Delta \mathbf{u}_{\mathbf{c}}^{\Sigma}+ \\
& \left(0.5 u_{c 00}^{\Sigma}\right)_{G_{i 0 M d c}} \Delta M_{d c}
\end{aligned}
$$

The small-signal models for PLL, signal filters and CCSC are the same as those for HB-MMC. Difference mainly exists in the modeling of ACC and DCC. The average capacitor voltage is regulated in ACC and the DC voltage is regulated in DCC, the corresponding small-signal models should be modified.

For ACC output, referring to (A20), $\Delta \mathbf{M}_{\mathbf{d q}}$ can be obtained:

$$
\begin{aligned}
\Delta \mathbf{M}_{\mathrm{dq}} & =\mathbf{G}_{\mathrm{Mdquxy}} \Delta \mathbf{u}_{\mathrm{pxy}}+\mathbf{G}_{\mathrm{Mdqixy}} \Delta \mathbf{i}_{\mathrm{xy}}+ \\
& \left(\left[\begin{array}{c}
G_{\text {in }} G_{\text {out }} \\
0
\end{array}\right] D_{\text {uc }}\left[\mathbf{0}_{1 \times 4} 1\right]\right)_{\mathbf{G}_{\text {Mdque }}} \Delta \mathbf{u}_{\mathrm{c}}^{\mathbf{2}}
\end{aligned}
$$

where $D_{u c}$ is the transfer function for signal filter of the average capacitor voltage. Similar to (A28), $\Delta \mathbf{M}_{\mathbf{x y}}$ can be further expressed as:

$$
\Delta \mathbf{M}_{\mathrm{xy}}=\mathbf{G}_{\mathrm{Mxyu}} \Delta \mathbf{u}_{\mathrm{pxy}}+\mathbf{G}_{\mathrm{Mxyi}} \Delta \mathbf{i}_{\mathrm{xy}}+\left(\mathbf{C}_{\mathbf{\theta 3}} \mathbf{G}_{\mathrm{Mdquc}}\right)_{\mathbf{G}_{\mathrm{Mxyuc}}} \Delta \mathbf{u}_{\mathrm{c}}^{\Sigma}
$$

The small-signal model of DCC output is:

$$
\begin{aligned}
\Delta M_{d c}= & {\left[\left(K_{\text {Udcs }}-G_{d c i n} G_{d c o u t}\right) D_{u d c}\right]_{G_{\text {Mdculc }}} \Delta u_{d c}+} \\
& \left(-3 G_{d c i n} D_{\text {idc }}\right)_{G_{\text {Mdci }}} \Delta i_{d i f f 0} 0
\end{aligned}
$$

where $G_{d c i n}=K_{P I d c}+K_{I I d c} / s, G_{d c o u t}=K_{\text {POdc }}+K_{\text {IOdc }} / s . D_{\text {udc }}$ and $D_{i d c}$ are the transfer functions of signal filters for the DC voltage and DC current.

Referring to (A34), the group of algebra equations should be modified as (A43). The difference between (A34) and (A43) is highlighted.

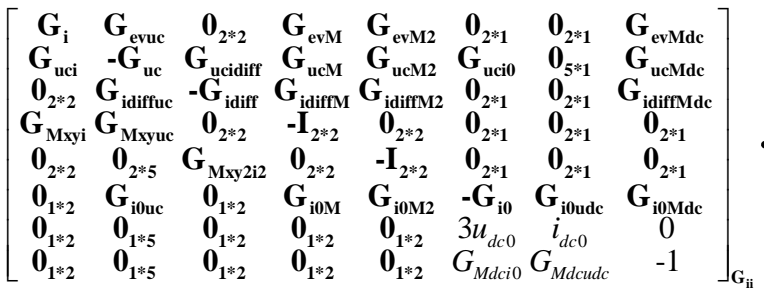

$$
\begin{aligned}
& {\left[\begin{array}{c}
\Delta \mathbf{i}_{\mathrm{xy}} \\
\Delta \mathbf{u}_{\mathrm{c}}^{2} \\
\Delta \mathbf{i}_{\text {diffry2 }}^{2} \\
\Delta \mathbf{M}_{\mathrm{xy}} \\
\Delta \mathbf{M}_{\mathrm{xy2}} \\
\Delta i_{d i f f 0} \\
\Delta u_{d c} \\
\Delta M_{d c}
\end{array}\right]=\left[\begin{array}{c}
\mathbf{I}_{2^{* 2}} / K_{T} \\
\mathbf{0}_{\mathbf{5}^{* 2}} \\
\mathbf{0}_{2^{* 2}} \\
-\mathbf{G}_{\mathrm{Mxyu}} \\
-\mathbf{G}_{\mathrm{Mxy2u}} \\
\mathbf{0}_{\mathbf{1}^{* 2}} \\
\mathbf{0}_{\mathbf{0}^{* 2}} \\
\mathbf{0}_{\mathbf{1}^{* 2}}
\end{array}\right]_{\mathbf{G}_{\mathrm{uu}}} \quad \Delta \mathbf{u}_{\mathrm{pxy}}}
\end{aligned}
$$

The expression of the AC impedance for the HY-MMC is the same as (A35).

TABLE AI

PARAMETERS OF THE TESTED SYSTEM

\begin{tabular}{lll}
\hline \hline & Rated DC voltage & $640 \mathrm{kV}$ \\
& Rated capacity & $1000 \mathrm{MW}$ \\
Half-bridge & Arm inductance & $112 \mathrm{mH}$ \\
/Hybrid & Arm equivalent resistance & $0.01 \Omega$ \\
MMC & Number of FBSM/HBSM (HY-MMC) & $100 / 100$ \\
& Number of HBSM (HB-MMC) & 200 \\
& Submodule capacitance & $10 \mathrm{mF}$ \\
\hline \multirow{2}{*}{ Two-level } & Rated DC voltage & $640 \mathrm{kV}$ \\
VSC & Rated capacity & $1000 \mathrm{MW}$ \\
& DC capacitance & $0.3 \mathrm{mF}$ \\
\hline AC & Rated voltage grid/converter-side & $400 \mathrm{kV} / 352 \mathrm{kV}$ \\
transformer & Leak inductance & $15 \%$ \\
\hline & Rated voltage & $400 \mathrm{kV}$ \\
AC system & Impedance (AC system 1, SCR=2) & $80 \angle 85.62^{\circ} \Omega$ \\
& Impedance (AC system $2, \mathrm{SCR}=20)$ & $8 \angle 82.83^{\circ} \Omega$ \\
\hline \hline
\end{tabular}

TABLE AII

CONTROL PARAMETERS OF THE CONVERTERS

\begin{tabular}{crc}
\hline \hline & Control parameters & VSCs \\
\hline PLL & $K_{P \text { pll }}$ & 80
\end{tabular}




\begin{tabular}{ccc} 
& $K_{\text {Ipll }}$ & 500 \\
\hline \multirow{4}{*}{ ACC } & $K_{P O}$ & $1($ Inv.) $0.4($ Rec. $)$ \\
& $K_{I O}$ & $100($ Inv. $) 300$ (Rec.) \\
& $K_{P I}$ & 1 \\
& $K_{I I}$ & 50 \\
\hline & $K_{\text {POdc }}$ & 1.2 \\
DCC (for & $K_{I O d c}$ & 800 \\
HY-MMC & $K_{P I d c}$ & 2 \\
only) & $K_{\text {IIdc }}$ & 100 \\
& $K_{U d c s}$ & 1 \\
\hline Cutting-off & active/reactive power & $800 \mathrm{~Hz}$ \\
frequencies & AC voltage & $300 \mathrm{~Hz}$ \\
for second- & AC current & $800 \mathrm{~Hz}$ \\
order low- & DC voltage & $300 \mathrm{~Hz}$ \\
pass signal & DC current & $800 \mathrm{~Hz}$ \\
filters & Sub-module capacitor voltage & $500 \mathrm{~Hz}$ \\
\hline \hline
\end{tabular}

\section{REFERENCES}

[1] N. Flourentzou, V. G. Agelidis and G. D. Demetriades, "VSC-Based HVDC Power Transmission Systems: An Overview," IEEE Trans. Power Electron., vol. 24, no. 3, pp. 592-602, March 2009.

[2] A. Lesnicar and R. Marquardt, "An innovative modular multilevel converter topology suitable for a wide power range," Proc. IEEE Power Tech Conf.,, Bologna, Italy, 2003, pp. 1-6

[3] R. Zeng, L. Xu, L. Yao and B. W. Williams, "Design and Operation of a Hybrid Modular Multilevel Converter," IEEE Trans. Power Electron., vol. 30, no. 3, pp. 1137-1146, March 2015.

[4] S. Yang, W. Xiang, R. Li, et al., "An Improved DC fault Protection Algorithm for MMC HVDC Grids based on Modal Domain Analysis," IEEE J. Emerg. Sel. Top. Power Electron., early access, to be published.

[5] W. Xiang, S. Yang, L. Xu, et al., "A Transient Voltage based DC Fault Line Protection Scheme for MMC based DC Grid Embedding DC Breakers," IEEE Trans. Power Del, vol. 34, no. 1, pp. 334-345, Feb. 2019.

[6] W. Lin, D. Jovcic, S. Nguefeu and H. Saad, "Full-Bridge MMC Converter Optimal Design to HVDC Operational Requirements," IEEE Trans. Power Del., vol. 31, no. 3, pp. 1342-1350, June 2016.

[7] W. Xiang, W. Lin, L. Xu and J. Wen, "Enhanced Independent Pole Control of Hybrid MMC-HVdc System," IEEE Trans. Power Del., vol. 33, no. 2, pp. 861-872, April 2018.

[8] Y. Huang, X. Yuan, J. Hu, P. Zhou and D. Wang, "DC-Bus Voltage Control Stability Affected by AC-Bus Voltage Control in VSCs Connected to Weak AC Grids," IEEE J. Emerg. Sel. Top. Power Electron., vol. 4, no. 2, pp. 445-458, June 2016.

[9] D. Wang, L. Liang, L. Shi, J. Hu and Y. Hou, "Analysis of Modal Resonance Between PLL and DC-Link Voltage Control in Weak-Grid Tied VSCs," IEEE Trans. Power Syst., vol. 34, no. 2, pp. 1127-1138, March 2019.

[10] J. Khazaei, M. Beza and M. Bongiorno, "Impedance Analysis of Modular Multi-Level Converters Connected to Weak AC Grids," IEEE Trans. Power Syst., vol. 33, no. 4, pp. 4015-4025, July 2018.

[11] B. Wen, D. Boroyevich, R. Burgos, P. Mattavelli and Z. Shen, "SmallSignal Stability Analysis of Three-Phase AC Systems in the Presence of Constant Power Loads Based on Measured d-q Frame Impedances," IEEE Trans. Power Electron., vol. 30, no. 10, pp. 5952-5963, Oct. 2015.

[12] B. Wen, D. Boroyevich, R. Burgos, P. Mattavelli and Z. Shen, "Analysis of D-Q Small-Signal Impedance of Grid-Tied Inverters," IEEE Trans. Power Electron, vol. 31, no. 1, pp. 675-687, Jan. 2016.

[13] B. Wen, D. Dong, D. Boroyevich, R. and et al., "Impedance-Based Analysis of Grid-Synchronization Stability for Three-Phase Paralleled Converters," IEEE Trans. Power Electron, vol. 31, no. 1, pp. 26-38, Jan. 2016.

[14] Y. Li, G. Tang, T. An, and et al., "Power Compensation Control for Interconnection of Weak Power Systems by VSC-HVDC," IEEE Trans. Power Del., vol. 32, no. 4, pp. 1964-1974, Aug. 2017.

[15] C. Zou, H. Rao, S. Xu, et al., "Analysis of Resonance Between a VSCHVDC Converter and the AC Grid," IEEE Trans. Power Electron., vol. 33, no. 12, pp. 10157-10168, Dec. 2018.

[16] X. Lu, W. Xiang and J. Wen, "Enhancing Active Power Transfer Capability for Hybrid MMC Integrated with Weak AC Grid through
Parameter Adjustment," Proc. 45th Annu. Conf. IEEE Ind. Electron. Soc., Lisbon, Portugal, 2019, pp. 4805-4810.

[17] J. Beerten, G. B. Diaz, S. D'Arco and J. A. Suul, "Comparison of smallsignal dynamics in MMC and two-level VSC HVDC transmission schemes," in Proc. IEEE Int. Energy Conf., Leuven, 2016, pp. 1-6.

[18] X. Lu, W. Xiang, W. Lin, J. Wen. "State-space model and PQ operation zone analysis of hybrid MMC," Electr. Power Syst. Res., vol. 162, pp. 99108, September 2018.

[19] X. Lu, W. Xiang, W. Lin, J. Wen, "Small-signal modeling of MMC based DC grid and analysis of the impact of DC reactors on the small-signal stability," Int. J. Electr. Power Energy Syst. vol. 101, pp. 25-37, October 2018.

[20] A. Jamshidifar and D. Jovcic, "Small-Signal Dynamic DQ Model of Modular Multilevel Converter for System Studies," IEEE Trans. Power Del., vol. 31, no. 1, pp. 191-199, Feb. 2016.

[21] J. Freytes et al., "Improving Small-Signal Stability of an MMC With CCSC by Control of the Internally Stored Energy," IEEE Trans. Power Del., vol. 33, no. 1, pp. 429-439, Feb. 2018.

[22] P. Kundur. "Power System Stability and Control," McGraw-Hill, 1994.

[23] S. Shah and L. Parsa, "Impedance Modeling of Three-Phase Voltage Source Converters in DQ, Sequence, and Phasor Domains," IEEE Trans. Energy Convers., vol. 32, no. 3, pp. 1139-1150, Sept. 2017.

[24] J. Z. Zhou, A. M. Gole. "Rationalisation and validation of dc power transfer limits for voltage sourced converter based high voltage DC transmission", IET Gener. Transm. Distrib., vol. 10, no. 6, pp. 1327-1335, May, 2016.

[25] G. Wu et al., "Analysis and design of vector control for VSC-HVDC connected to weak grids," CSEE J. Power Energy Syst., vol. 3, no. 2, pp. 115-124, June 2017.

[26] M. F. M. Arani and Y. A. I. Mohamed, "Analysis and Performance Enhancement of Vector-Controlled VSC in HVDC Links Connected to Very Weak Grids," IEEE Trans. Power Syst., vol. 32, no. 1, pp. 684-693, Jan. 2017.

[27] Y. Huang and D. Wang, "Effect of Control-Loops Interactions on Power Stability Limits of VSC Integrated to AC System," IEEE Trans. Power Del., vol. 33, no. 1, pp. 301-310, Feb. 2018.

[28] A. J. Agbemuko, J. L. Dominguez-Garcia, O. Gomis-Bellmunt and L. Harnefors, "Passivity-Based Analysis and Performance Enhancement of a Vector Controlled VSC Connected to a Weak AC Grid," IEEE Trans Power Del. Early access, to be published.

\section{BIOGRAPHIES}

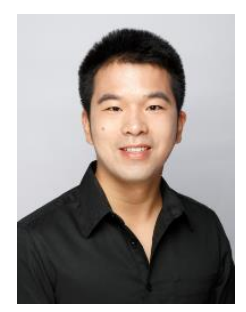

Xiaojun Lu received his B.Eng. and PhD degrees both in electrical engineering from Huazhong University of Science and Technology (HUST), China in 2013 and 2018, respectively. Currently, he is a postdoctor with HUST since 2018. His main research interests include modeling and stability analysis of MMC-HVDC and dc grids.

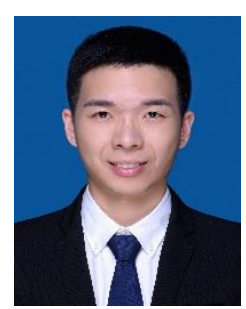

Wang Xiang (S'16-M'17) received his B.Eng. and $\mathrm{PhD}$ degrees both in electrical engineering from Huazhong University of Science and Technology (HUST), China in 2012 and 2017, respectively. He was a visiting student at the University of Aberdeen and the University of Strathclyde in 2014 and 2016 respectively. Currently, he is a research associate with the University of Strathclyde since 2018. His main research interests include MMCHVDC, high power dc/dc converters and de grids. 


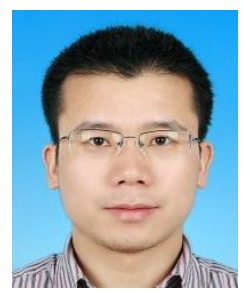

Weixing Lin (M'13) obtained his B.E. and PhD degrees in electrical engineering from Huazhong University of Science and Technology (HUST), China, in 2008 and 2014 respectively. He was a research fellow at University of Aberdeen during 2012-2016. He is currently the chief engineer of HVDC division at TBEA China Xinjiang Sunoasis Co. Ltd. His research interests are HVDC, MMC, dc-dc autotransformer, DC grids.

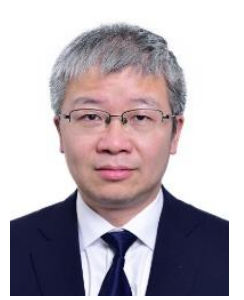

Jinyu Wen (M'10) received his B.Eng. and Ph.D. degrees all in electrical engineering from Huazhong University of Science and Technology (HUST), Wuhan, China, in 1992 and 1998, respectively. He was a visiting student from 1996 to 1997 and a research fellow from 2002 to 2003 all at the University of Liverpool, UK, and a senior visiting researcher at the University of Texas at Arlington, USA in 2010. From 1998 to 2002 he was a director engineer in XJ Electric Co. Ltd. in China. In 2003 he joined the HUST and now is a Professor at HUST. His current research interests include renewable energy integration, energy storage application, DC grid, and power system operation and control. 